\title{
Analysis and prediction of pathways in HeLa cells by integrating biological levels of organization with systems-biology approaches
}

\author{
Supplemental Material
}

\begin{tabular}{|l|l|l|}
\hline Location & Pathway size & Genes present \\
\hline GO:0005737 cytoplasm & 8475 & $8253(97.4 \%)$ \\
\hline GO:0044444 cytoplasmic part & 6092 & $5961(97.9 \%)$ \\
\hline GO:0043231 intracellular membrane-bounded organelle & 8980 & $8704(97.0 \%)$ \\
\hline GO:0070013 intracellular organelle lumen & 2668 & $2621(98.3 \%)$ \\
\hline GO:0044428 nuclear part & 2594 & $2543(98.1 \%)$ \\
\hline GO:0005626 insoluble fraction & 904 & $896(99.1 \%)$ \\
\hline $\begin{array}{l}\text { GO:0043232 intracellular non-membrane-bounded } \\
\text { organelle }\end{array}$ & 3310 & $3225(97.5 \%)$ \\
\hline GO:0031967 organelle envelope & 751 & $740(98.5 \%)$ \\
\hline GO:0044459 plasma membrane part & 1958 & $1908(97.4 \%)$ \\
\hline GO:0005625 soluble fraction & 395 & $392(99.2 \%)$ \\
\hline GO:0044429 mitochondrial part & 705 & $694(98.4 \%)$ \\
\hline GO:0044430 cytoskeletal part & 1153 & $1127(97.8 \%)$ \\
\hline GO:0044432 endoplasmic reticulum part & 770 & $756(98.2 \%)$ \\
\hline GO:0044431 Golgi apparatus part & 597 & $587(98.3 \%)$ \\
\hline GO:0043005 neuron projection & 522 & $514(98.5 \%)$ \\
\hline GO:0031988 membrane-bounded vesicle & 758 & $743(98.0 \%)$ \\
\hline GO:0031410 cytoplasmic vesicle & 784 & $768(98.0 \%)$ \\
\hline $\begin{array}{l}\text { GO:0042175 nuclear outer membrane-endoplasmic } \\
\text { reticulum membrane network }\end{array}$ & 697 & $683(98.0 \%)$ \\
\hline GO:0044437 vacuolar part & 172 & $171(99.4 \%)$ \\
\hline GO:0031300 intrinsic to organelle membrane & 167 & $166(99.4 \%)$ \\
\hline GO:0044440 endosomal part & 275 & $271(98.5 \%)$ \\
\hline GO:0016585 chromatin remodeling complex & 109 & $109(100.0 \%)$ \\
\hline
\end{tabular}

Table S1 Enriched gene ontology level 3 categories of biological processes of total expression analysis. Set size refers to the number of entities that have a UniProt ID as stated in the corresponding GO category at the ConsensusPathDB site. The number of candidates contained refers to amount of proteins identified in this study that appear as part of the GO category. P-values are calculated according to a hypergeometric test; q-values represent $p$-values corrected for multiple testing using the false discovery rate method. It is worth noting that the expressions of each GO term fulfill at least $97 \%$ of the total reported for each term. 


\begin{tabular}{|c|c|c|c|c|}
\hline Gene Ontology term & $\begin{array}{l}\text { Set } \\
\text { size }\end{array}$ & $\begin{array}{l}\text { Candidates } \\
\text { contained }\end{array}$ & P-value & Q-value \\
\hline GO:0022403 cell cycle phase & 772 & $309(40.0 \%)$ & 1.29E-53 & $7.15 \mathrm{E}-51$ \\
\hline GO:0000278 mitotic cell cycle & 687 & $261(38.0 \%)$ & $2.21 \mathrm{E}-40$ & $6.12 \mathrm{E}-38$ \\
\hline $\begin{array}{l}\text { GO:0006139 nucleobase, nucleoside, nucleotide and nucleic acid } \\
\text { metabolic process }\end{array}$ & 4958 & $1120(22.6 \%)$ & 8.03E-32 & $1.48 \mathrm{E}-29$ \\
\hline GO:0034641 cellular nitrogen compound metabolic process & 5373 & $1194(22.2 \%)$ & $3.38 \mathrm{E}-31$ & $4.68 \mathrm{E}-29$ \\
\hline GO:0071842 cellular component organization at cellular level & 2857 & $704(24.7 \%)$ & $4.98 \mathrm{E}-29$ & $5.52 \mathrm{E}-27$ \\
\hline GO:0044260 cellular macromolecule metabolic process & 6149 & $1322(21.5 \%)$ & $2.01 \mathrm{E}-28$ & $1.85 \mathrm{E}-26$ \\
\hline GO:0033554 cellular response to stress & 957 & $262(27.4 \%)$ & $2.86 \mathrm{E}-16$ & $2.26 \mathrm{E}-14$ \\
\hline GO:0051321 meiotic cell cycle & 138 & $62(44.9 \%)$ & $2.48 \mathrm{E}-14$ & $1.72 \mathrm{E}-12$ \\
\hline GO:0007050 cell cycle arrest & 335 & $113(33.7 \%)$ & $9.40 \mathrm{E}-14$ & $5.79 \mathrm{E}-12$ \\
\hline GO:0009059 macromolecule biosynthetic process & 3775 & $798(21.2 \%)$ & $4.45 \mathrm{E}-13$ & $2.46 \mathrm{E}-11$ \\
\hline GO:0044249 cellular biosynthetic process & 4603 & $949(20.6 \%)$ & $5.99 \mathrm{E}-13$ & $3.02 \mathrm{E}-11$ \\
\hline GO:0043933 macromolecular complex subunit organization & 1008 & $254(25.2 \%)$ & $1.66 \mathrm{E}-11$ & $7.65 \mathrm{E}-10$ \\
\hline GO:0010467 gene expression & 3914 & $806(20.6 \%)$ & $1.26 \mathrm{E}-10$ & 5.37E-09 \\
\hline GO:0019222 regulation of metabolic process & 4485 & $907(20.2 \%)$ & $3.22 \mathrm{E}-10$ & $1.28 \mathrm{E}-08$ \\
\hline GO:0000226 microtubule cytoskeleton organization & 215 & $73(34.0 \%)$ & $1.60 \mathrm{E}-09$ & 5.89E-08 \\
\hline GO:0000086 G2/M transition of mitotic cell cycle & 135 & $52(38.5 \%)$ & $2.72 \mathrm{E}-09$ & $9.13 \mathrm{E}-08$ \\
\hline GO:0022607 cellular component assembly & 1282 & $299(23.3 \%)$ & $2.80 \mathrm{E}-09$ & $9.13 \mathrm{E}-08$ \\
\hline GO:0048519 negative regulation of biological process & 2583 & $548(21.2 \%)$ & 4.57E-09 & $1.41 \mathrm{E}-07$ \\
\hline GO:0050794 regulation of cellular process & 7306 & $1393(19.1 \%)$ & $1.21 \mathrm{E}-08$ & $3.53 \mathrm{E}-07$ \\
\hline GO:0000819 sister chromatid segregation & 45 & $24(53.3 \%)$ & $3.54 \mathrm{E}-08$ & $9.82 \mathrm{E}-07$ \\
\hline GO:0007062 sister chromatid cohesion & 26 & $17(65.4 \%)$ & $6.04 \mathrm{E}-08$ & $1.59 \mathrm{E}-06$ \\
\hline GO:0007091 mitotic metaphase/anaphase transition & 43 & $22(51.2 \%)$ & $3.45 \mathrm{E}-07$ & $8.68 \mathrm{E}-06$ \\
\hline $\begin{array}{l}\text { GO:0000083 regulation of transcription involved in G1/S phase } \\
\text { of mitotic cell cycle }\end{array}$ & 21 & $14(66.7 \%)$ & $6.49 \mathrm{E}-07$ & $1.53 \mathrm{E}-05$ \\
\hline GO:0007131 reciprocal meiotic recombination & 35 & $19(54.3 \%)$ & $6.62 \mathrm{E}-07$ & $1.53 \mathrm{E}-05$ \\
\hline GO:0043412 macromolecule modification & 2212 & $458(20.7 \%)$ & $2.16 \mathrm{E}-06$ & 4.79E-05 \\
\hline GO:0000082 G1/S transition of mitotic cell cycle & 169 & $53(31.4 \%)$ & $4.13 \mathrm{E}-06$ & $8.80 \mathrm{E}-05$ \\
\hline GO:0006725 cellular aromatic compound metabolic process & 223 & $65(29.1 \%)$ & $5.88 \mathrm{E}-06$ & 0.000121 \\
\hline GO:0007098 centrosome cycle & 31 & $16(51.6 \%)$ & $1.20 \mathrm{E}-05$ & 0.000238 \\
\hline GO:0050658 RNA transport & 127 & $41(32.3 \%)$ & $2.27 \mathrm{E}-05$ & 0.000435 \\
\hline GO:0006403 RNA localization & 132 & $42(31.8 \%)$ & 2.69E-05 & 0.000483 \\
\hline $\begin{array}{l}\text { GO:0015931 nucleobase, nucleoside, nucleotide and nucleic acid } \\
\text { transport }\end{array}$ & 149 & 46 (30.9\%) & $2.70 \mathrm{E}-05$ & 0.000483 \\
\hline GO:0000216 M/G1 transition of mitotic cell cycle & 79 & $28(35.4 \%)$ & 7.04E-05 & 0.00122 \\
\hline
\end{tabular}

Table S2 Enriched gene ontology level 3 categories of biological processes of over-expressed genes. Set size refers to the number of entities that have a UniProt ID as stated in the corresponding GO category at the ConsensusPathDB site. The number of candidates contained refers to amount of proteins identified in this study that appear as part of the GO category. P-values are calculated according to a hypergeometric test; q-values represent $p$-values corrected for multiple testing using the false discovery rate method. 


\begin{tabular}{|c|c|c|c|c|}
\hline Gene Ontology term & $\begin{array}{l}\text { Set } \\
\text { size }\end{array}$ & $\begin{array}{l}\text { Candidates } \\
\text { contained }\end{array}$ & P-value & Q-value \\
\hline GO:0022403 cell cycle phase & 772 & $257(33.3 \%)$ & $5.33 \mathrm{E}-49$ & $2.86 \mathrm{E}-46$ \\
\hline GO:0034641 cellular nitrogen compound metabolic process & 5373 & $1002(18.7 \%)$ & $7.02 E-43$ & $1.88 \mathrm{E}-40$ \\
\hline $\begin{array}{l}\text { GO:0006139 nucleobase, nucleoside, nucleotide and } \\
\text { nucleic acid metabolic process }\end{array}$ & 4958 & $936(18.9 \%)$ & $2.23 \mathrm{E}-41$ & $3.98 \mathrm{E}-39$ \\
\hline GO:0044260 cellular macromolecule metabolic process & 6149 & 1098 (17.9\%) & $6.4 \mathrm{E}-39$ & $7.59 \mathrm{E}-37$ \\
\hline GO:0000278 mitotic cell cycle & 687 & $220(32.0 \%)$ & $7.08 \mathrm{E}-39$ & $7.59 \mathrm{E}-37$ \\
\hline $\begin{array}{l}\text { GO:0071842 cellular component organization at cellular } \\
\text { level }\end{array}$ & 2857 & $602(21.1 \%)$ & $1.04 \mathrm{E}-37$ & $9.33 \mathrm{E}-36$ \\
\hline GO:0044249 cellular biosynthetic process & 4603 & $809(17.6 \%)$ & $3.4 \mathrm{E}-23$ & $2.6 \mathrm{E}-21$ \\
\hline GO:0033554 cellular response to stress & 957 & $234(24.5 \%)$ & $2.68 \mathrm{E}-22$ & $1.8 \mathrm{E}-20$ \\
\hline GO:0009059 macromolecule biosynthetic process & 3775 & $669(17.7 \%)$ & $2.83 \mathrm{E}-19$ & $1.68 \mathrm{E}-17$ \\
\hline GO:0019222 regulation of metabolic process & 4485 & $766(17.1 \%)$ & 4.77E-18 & $2.56 \mathrm{E}-16$ \\
\hline GO:0050794 regulation of cellular process & 7306 & $1156(15.8 \%)$ & $1.45 \mathrm{E}-17$ & $7.08 \mathrm{E}-16$ \\
\hline GO:0043933 macromolecular complex subunit organization & 1008 & $225(22.4 \%)$ & $1.81 \mathrm{E}-16$ & $8.08 \mathrm{E}-15$ \\
\hline GO:0010467 gene expression & 3914 & $667(17.1 \%)$ & $4.19 \mathrm{E}-15$ & $1.73 \mathrm{E}-13$ \\
\hline GO:0007050 cell cycle arrest & 335 & $98(29.3 \%)$ & $6.28 \mathrm{E}-15$ & $2.4 \mathrm{E}-13$ \\
\hline GO:0048519 negative regulation of biological process & 2583 & $469(18.2 \%)$ & $8.27 \mathrm{E}-15$ & $2.95 \mathrm{E}-13$ \\
\hline GO:0022607 cellular component assembly & 1282 & $258(20.1 \%)$ & $5.03 \mathrm{E}-13$ & $1.68 \mathrm{E}-11$ \\
\hline GO:0051321 meiotic cell cycle & 138 & $51(37.0 \%)$ & 1.49E-12 & 4.69E-11 \\
\hline GO:0000086 G2/M transition of mitotic cell cycle & 135 & $49(36.3 \%)$ & $8.75 \mathrm{E}-12$ & $2.6 \mathrm{E}-10$ \\
\hline GO:0048518 positive regulation of biological process & 2857 & $493(17.3 \%)$ & $1.03 \mathrm{E}-11$ & $2.92 \mathrm{E}-10$ \\
\hline GO:0043412 macromolecule modification & 2212 & $384(17.4 \%)$ & $1.8 \mathrm{E}-09$ & $4.84 \mathrm{E}-08$ \\
\hline GO:0042592 homeostatic process & 996 & $196(19.7 \%)$ & $2.94 \mathrm{E}-09$ & 0.000000075 \\
\hline GO:0048731 system development & 3031 & $501(16.5 \%)$ & 5.11E-09 & 0.000000125 \\
\hline GO:0000819 sister chromatid segregation & 45 & $22(48.9 \%)$ & $8.2 \mathrm{E}-09$ & 0.000000191 \\
\hline GO:0007131 reciprocal meiotic recombination & 35 & $19(54.3 \%)$ & 9.23E-09 & 0.000000206 \\
\hline GO:0019538 protein metabolic process & 3414 & $553(16.2 \%)$ & $1.36 \mathrm{E}-08$ & 0.000000291 \\
\hline GO:0048513 organ development & 2079 & $358(17.2 \%)$ & $1.75 \mathrm{E}-08$ & 0.00000036 \\
\hline GO:0000226 microtubule cytoskeleton organization & 215 & $59(27.4 \%)$ & 2.09E-08 & 0.000000415 \\
\hline GO:0007091 mitotic metaphase/anaphase transition & 43 & $20(46.5 \%)$ & 0.000000113 & 0.00000216 \\
\hline GO:0030154 cell differentiation & 2273 & $379(16.7 \%)$ & 0.000000256 & 0.00000473 \\
\hline GO:0009725 response to hormone stimulus & 660 & $132(20.0 \%)$ & 0.000000492 & 0.00000879 \\
\hline GO:0051128 regulation of cellular component organization & 935 & $175(18.7 \%)$ & 0.000000733 & 0.0000127 \\
\hline GO:0010033 response to organic substance & 1394 & $245(17.6 \%)$ & 0.000000895 & 0.000015 \\
\hline GO:0001666 response to hypoxia & 203 & $52(25.6 \%)$ & 0.00000143 & 0.0000233 \\
\hline GO:0070482 response to oxygen levels & 221 & $55(24.9 \%)$ & 0.00000191 & 0.0000301 \\
\hline GO:0006793 phosphorus metabolic process & 1306 & $228(17.5 \%)$ & 0.00000363 & 0.0000556 \\
\hline GO:0070887 cellular response to chemical stimulus & 1112 & $197(17.7 \%)$ & 0.00000674 & 0.0001 \\
\hline GO:0050793 regulation of developmental process & 1066 & $189(17.7 \%)$ & 0.00000994 & 0.000144 \\
\hline GO:0070271 protein complex biogenesis & 604 & $117(19.4 \%)$ & 0.0000106 & 0.000149 \\
\hline $\begin{array}{l}\text { GO:0006725 cellular aromatic compound metabolic } \\
\text { process }\end{array}$ & 223 & $53(23.8 \%)$ & 0.0000122 & 0.000167 \\
\hline
\end{tabular}




\begin{tabular}{|c|c|c|c|c|}
\hline GO:0000082 G1/S transition of mitotic cell cycle & 169 & $43(25.4 \%)$ & 0.0000135 & 0.000181 \\
\hline GO:0007599 hemostasis & 512 & $101(19.7 \%)$ & 0.0000194 & 0.000254 \\
\hline $\begin{array}{l}\text { GO:0009792 embryo development ending in birth or egg } \\
\text { hatching }\end{array}$ & 399 & $82(20.6 \%)$ & 0.000026 & 0.000331 \\
\hline GO:0007596 blood coagulation & 508 & $99(19.5 \%)$ & 0.0000387 & 0.000483 \\
\hline GO:0010035 response to inorganic substance & 332 & $70(21.1 \%)$ & 0.0000424 & 0.000507 \\
\hline GO:0042493 response to drug & 338 & $71(21.0 \%)$ & 0.0000425 & 0.000507 \\
\hline GO:0007062 sister chromatid cohesion & 26 & $12(46.2 \%)$ & 0.0000445 & 0.000518 \\
\hline GO:0012501 programmed cell death & 1390 & $233(16.8 \%)$ & 0.0000489 & 0.000557 \\
\hline GO:0009611 response to wounding & 1017 & $177(17.4 \%)$ & 0.0000554 & 0.000619 \\
\hline GO:0007098 centrosome cycle & 31 & $13(41.9 \%)$ & 0.0000738 & 0.000808 \\
\hline GO:0050658 RNA transport & 127 & $33(26.0 \%)$ & 0.0000836 & 0.000896 \\
\hline $\begin{array}{l}\text { GO:0000083 regulation of transcription involved in G1/S } \\
\text { phase of mitotic cell cycle }\end{array}$ & 21 & $10(47.6 \%)$ & 0.000141 & 0.00148 \\
\hline GO:0000216 M/G1 transition of mitotic cell cycle & 79 & $23(29.1 \%)$ & 0.000158 & 0.0016 \\
\hline GO:0007052 mitotic spindle organization & 25 & $11(44.0 \%)$ & 0.000158 & 0.0016 \\
\hline GO:0006403 RNA localization & 132 & $33(25.0 \%)$ & 0.000185 & 0.00184 \\
\hline $\begin{array}{l}\text { GO:0015931 nucleobase, nucleoside, nucleotide and } \\
\text { nucleic acid transport }\end{array}$ & 149 & $36(24.2 \%)$ & 0.000202 & 0.00197 \\
\hline GO:0001568 blood vessel development & 386 & $76(19.7 \%)$ & 0.000212 & 0.00202 \\
\hline GO:0048514 blood vessel morphogenesis & 339 & $67(19.8 \%)$ & 0.000436 & 0.0041 \\
\hline GO:0005975 carbohydrate metabolic process & 646 & $115(17.8 \%)$ & 0.000464 & 0.00429 \\
\hline GO:0051239 regulation of multicellular organismal process & 1402 & $227(16.2 \%)$ & 0.000497 & 0.00451 \\
\hline GO:0006959 humoral immune response & 112 & $28(25.0 \%)$ & 0.00055 & 0.00491 \\
\hline GO:0035295 tube development & 331 & 65 (19.6\%) & 0.000628 & 0.00552 \\
\hline GO:0048468 cell development & 1211 & $198(16.4 \%)$ & 0.000694 & 0.006 \\
\hline GO:0006979 response to oxidative stress & 223 & $47(21.1 \%)$ & 0.000724 & 0.00616 \\
\hline GO:0044093 positive regulation of molecular function & 982 & $164(16.7 \%)$ & 0.000772 & 0.00646 \\
\hline GO:0001525 angiogenesis & 285 & $57(20.0 \%)$ & 0.000834 & 0.00688 \\
\hline GO:0044283 small molecule biosynthetic process & 666 & $116(17.4 \%)$ & 0.000951 & 0.00772 \\
\hline $\begin{array}{l}\text { GO:0006091 generation of precursor metabolites and } \\
\text { energy }\end{array}$ & 427 & $79(18.5 \%)$ & 0.00113 & 0.009 \\
\hline GO:0009057 macromolecule catabolic process & 715 & $123(17.2 \%)$ & 0.00115 & 0.009 \\
\hline GO:0002237 response to molecule of bacterial origin & 186 & $40(21.5 \%)$ & 0.00116 & 0.009 \\
\hline $\begin{array}{l}\text { GO:0035821 modification of morphology or physiology of } \\
\text { other organism }\end{array}$ & 35 & $12(34.3 \%)$ & 0.00122 & 0.00936 \\
\hline GO:0042023 DNA endoreduplication & 5 & $4(80.0 \%)$ & 0.00137 & 0.0103 \\
\hline GO:0048469 cell maturation & 91 & $23(25.3 \%)$ & 0.00141 & 0.0105 \\
\hline GO:0007584 response to nutrient & 225 & $46(20.4 \%)$ & 0.00159 & 0.0115 \\
\hline GO:0009314 response to radiation & 268 & $53(19.8 \%)$ & 0.00161 & 0.0115 \\
\hline GO:0071495 cellular response to endogenous stimulus & 381 & $71(18.6 \%)$ & 0.00161 & 0.0115 \\
\hline GO:0007165 signal transduction & 3729 & $548(14.7 \%)$ & 0.00174 & 0.0122 \\
\hline GO:0040008 regulation of growth & 440 & $80(18.2 \%)$ & 0.00176 & 0.0122 \\
\hline GO:0021915 neural tube development & 99 & $24(24.2 \%)$ & 0.00207 & 0.0142 \\
\hline GO:0048659 smooth muscle cell proliferation & 62 & $17(27.4 \%)$ & 0.00225 & 0.0152 \\
\hline GO:0009636 response to toxin & 95 & $23(24.2 \%)$ & 0.00258 & 0.0173 \\
\hline
\end{tabular}




\begin{tabular}{|l|l|l|l|l|}
\hline GO:0051303 establishment of chromosome localization & 20 & $8(40.0 \%)$ & 0.00266 & 0.0174 \\
\hline GO:0060323 head morphogenesis & 20 & $8(40.0 \%)$ & 0.00266 & 0.0174 \\
\hline $\begin{array}{l}\text { GO:0042558 } \\
\text { process }\end{array}$ & 34 & $11(32.4 \%)$ & 0.00327 & 0.0211 \\
\hline GO:0046677 response to antibiotic & 30 & $10(33.3 \%)$ & 0.00389 & 0.0248 \\
\hline GO:0046148 pigment biosynthetic process & 45 & $13(28.9 \%)$ & 0.00438 & 0.0276 \\
\hline GO:0009991 response to extracellular stimulus & 351 & $64(18.2 \%)$ & 0.00446 & 0.0278 \\
\hline GO:0009887 organ morphogenesis & 660 & $110(16.7 \%)$ & 0.00568 & 0.035 \\
\hline GO:0051702 interaction with symbiont & 18 & $7(38.9 \%)$ & 0.00589 & 0.0356 \\
\hline GO:0045137 development of primary sexual characteristics & 203 & $40(19.7 \%)$ & 0.00597 & 0.0356 \\
\hline GO:0046483 heterocycle metabolic process & 1019 & $162(15.9 \%)$ & 0.00611 & 0.0356 \\
\hline GO:0001556 oocyte maturation & 14 & $6(42.9 \%)$ & 0.00618 & 0.0356 \\
\hline GO:0010573 vascular endothelial growth factor production & 14 & $6(42.9 \%)$ & 0.00618 & 0.0356 \\
\hline GO:0045132 meiotic chromosome segregation & 14 & $6(42.9 \%)$ & 0.00618 & 0.0356 \\
\hline GO:0006066 alcohol metabolic process & 521 & $89(17.1 \%)$ & 0.00628 & 0.0358 \\
\hline $\begin{array}{l}\text { GO:0055086 } \\
\text { metabolic process }\end{array}$ & 911 & $146(16.0 \%)$ & 0.00674 & 0.038 \\
\hline GO:0031647 regulation of protein stability & & & & \\
\hline GO:0007548 sex differentiation & 74 & $18(24.3 \%)$ & 0.00682 & 0.0381 \\
\hline GO:0032844 regulation of homeostatic process & 230 & $44(19.1 \%)$ & 0.00707 & 0.0391 \\
\hline GO:0044248 cellular catabolic process & 181 & $36(19.9 \%)$ & 0.00754 & 0.0412 \\
\hline GO:0007099 centriole replication & 1483 & $227(15.3 \%)$ & 0.00838 & 0.0453 \\
\hline GO:0048729 tissue morphogenesis & 11 & $5(45.5 \%)$ & 0.00932 & 0.05 \\
\hline GO:0042180 cellular ketone metabolic process & 350 & $62(17.7 \%)$ & 0.00953 & 0.0506 \\
\hline $\begin{array}{l}\text { GO:0044403 symbiosis, encompassing mutualism through } \\
\text { parasitism }\end{array}$ & 88 & $129(16.1 \%)$ & 0.00963 & 0.0506 \\
\hline
\end{tabular}

Table S3 Enriched gene ontology level 3 categories of biological processes of over-expressed-TFs networks. Set size refers to the number of entities that have a UniProt ID as stated in the corresponding GO category at the ConsensusPathDB site. The number of candidates contained refers to amount of proteins identified in this study that appear as part of the GO category. P-values are calculated according to a hypergeometric test; q-values represent $p$-values corrected for multiple testing using the false discovery rate method. 


\begin{tabular}{|c|c|c|c|c|}
\hline gene ontology term & $\begin{array}{l}\text { set } \\
\text { size }\end{array}$ & $\begin{array}{l}\text { candidates } \\
\text { contained }\end{array}$ & p-value & q-value \\
\hline GO:0048731 system development & 3031 & $415(13.7 \%)$ & $1.36 \mathrm{E}-26$ & 7.11E-24 \\
\hline GO:0009888 tissue development & 1010 & $181(17.9 \%)$ & $4.44 \mathrm{E}-23$ & $1.16 \mathrm{E}-20$ \\
\hline GO:0007165 signal transduction & 3729 & $459(12.3 \%)$ & $1.16 \mathrm{E}-19$ & $2.02 \mathrm{E}-17$ \\
\hline GO:0048513 organ development & 2079 & $291(14.0 \%)$ & $2.23 \mathrm{E}-19$ & $2.92 \mathrm{E}-17$ \\
\hline GO:0030154 cell differentiation & 2273 & $308(13.6 \%)$ & $2.14 \mathrm{E}-18$ & $2.23 \mathrm{E}-16$ \\
\hline $\begin{array}{l}\text { GO:0051239 regulation of multicellular organismal } \\
\text { process }\end{array}$ & 1402 & $205(14.6 \%)$ & $1.52 \mathrm{E}-15$ & $1.33 \mathrm{E}-13$ \\
\hline GO:0010033 response to organic substance & 1394 & $201(14.4 \%)$ & $1.28 \mathrm{E}-14$ & $9.56 \mathrm{E}-13$ \\
\hline GO:0032989 cellular component morphogenesis & 800 & $133(16.6 \%)$ & $1.96 \mathrm{E}-14$ & $1.28 \mathrm{E}-12$ \\
\hline GO:0048514 blood vessel morphogenesis & 339 & $74(21.8 \%)$ & $2.21 \mathrm{E}-14$ & $1.29 \mathrm{E}-12$ \\
\hline GO:0009611 response to wounding & 1017 & $158(15.5 \%)$ & $2.48 \mathrm{E}-14$ & $1.3 \mathrm{E}-12$ \\
\hline GO:0001525 angiogenesis & 285 & $65(22.8 \%)$ & $1.01 \mathrm{E}-13$ & $4.8 \mathrm{E}-12$ \\
\hline $\begin{array}{l}\text { GO:0051128 regulation of cellular component } \\
\text { organization }\end{array}$ & 935 & $146(15.6 \%)$ & $1.75 \mathrm{E}-13$ & $7.29 \mathrm{E}-12$ \\
\hline GO:0070887 cellular response to chemical stimulus & 1112 & $166(14.9 \%)$ & $1.81 \mathrm{E}-13$ & $7.29 \mathrm{E}-12$ \\
\hline GO:0001568 blood vessel development & 386 & $78(20.2 \%)$ & $3.31 \mathrm{E}-13$ & $1.24 \mathrm{E}-11$ \\
\hline GO:0048468 cell development & 1211 & $174(14.4 \%)$ & $1.35 \mathrm{E}-12$ & 4.69E-11 \\
\hline GO:0034329 cell junction assembly & 149 & $42(28.2 \%)$ & $1.63 \mathrm{E}-12$ & $5.34 \mathrm{E}-11$ \\
\hline GO:0048583 regulation of response to stimulus & 1870 & $242(12.9 \%)$ & $3.65 \mathrm{E}-12$ & $1.12 \mathrm{E}-10$ \\
\hline GO:0050794 regulation of cellular process & 7306 & $746(10.2 \%)$ & $5.3 \mathrm{E}-12$ & $1.54 \mathrm{E}-10$ \\
\hline GO:0023051 regulation of signaling & 1622 & $213(13.1 \%)$ & $2.39 \mathrm{E}-11$ & $6.57 \mathrm{E}-10$ \\
\hline GO:0032879 regulation of localization & 1009 & $146(14.5 \%)$ & $6.23 \mathrm{E}-11$ & $1.63 \mathrm{E}-09$ \\
\hline GO:0050793 regulation of developmental process & 1066 & $152(14.3 \%)$ & $7.35 \mathrm{E}-11$ & $1.74 \mathrm{E}-09$ \\
\hline GO:0048518 positive regulation of biological process & 2857 & $334(11.7 \%)$ & $7.43 \mathrm{E}-11$ & $1.74 \mathrm{E}-09$ \\
\hline GO:0006629 lipid metabolic process & 1030 & $148(14.4 \%)$ & $7.63 \mathrm{E}-11$ & $1.74 \mathrm{E}-09$ \\
\hline GO:0048519 negative regulation of biological process & 2583 & $303(11.7 \%)$ & $5.29 \mathrm{E}-10$ & $1.15 \mathrm{E}-08$ \\
\hline GO:0040012 regulation of locomotion & 315 & $61(19.4 \%)$ & $7.63 \mathrm{E}-10$ & 0.000000016 \\
\hline GO:0009887 organ morphogenesis & 660 & $103(15.6 \%)$ & $8.16 \mathrm{E}-10$ & $1.64 \mathrm{E}-08$ \\
\hline GO:0048729 tissue morphogenesis & 350 & $65(18.6 \%)$ & $1.28 \mathrm{E}-09$ & 2.49E-08 \\
\hline GO:0045216 cell-cell junction organization & 100 & $28(28.0 \%)$ & $9.7 \mathrm{E}-09$ & 0.000000181 \\
\hline GO:0046903 secretion & 716 & $103(14.4 \%)$ & $6.44 \mathrm{E}-08$ & 0.00000116 \\
\hline GO:0012501 programmed cell death & 1390 & $174(12.5 \%)$ & $6.82 \mathrm{E}-08$ & 0.00000119 \\
\hline GO:0022612 gland morphogenesis & 90 & $25(27.8 \%)$ & 7.07E-08 & 0.00000119 \\
\hline GO:0030036 actin cytoskeleton organization & 337 & $58(17.2 \%)$ & 0.000000159 & 0.0000026 \\
\hline GO:0035295 tube development & 331 & $57(17.2 \%)$ & 0.000000199 & 0.00000316 \\
\hline GO:0002682 regulation of immune system process & 748 & $103(13.8 \%)$ & 0.000000555 & 0.00000854 \\
\hline GO:0030168 platelet activation & 246 & $45(18.3 \%)$ & 0.000000683 & 0.0000102 \\
\hline GO:0044255 cellular lipid metabolic process & 706 & $98(13.9 \%)$ & 0.000000729 & 0.0000106 \\
\hline GO:0002237 response to molecule of bacterial origin & 186 & $37(19.9 \%)$ & 0.000000822 & 0.0000116 \\
\hline GO:0010035 response to inorganic substance & 332 & $55(16.6 \%)$ & 0.00000115 & 0.0000159 \\
\hline $\begin{array}{l}\text { GO:0071842 cellular component organization at } \\
\text { cellular level }\end{array}$ & 2857 & $309(10.8 \%)$ & 0.00000125 & 0.0000168 \\
\hline GO:0061024 membrane organization & 552 & 80 (14.5\%) & 0.00000145 & 0.0000185 \\
\hline GO:0070482 response to oxygen levels & 221 & $41(18.6 \%)$ & 0.00000145 & 0.0000185 \\
\hline
\end{tabular}




\begin{tabular}{|c|c|c|c|c|}
\hline GO:0008610 lipid biosynthetic process & 448 & $68(15.2 \%)$ & 0.00000176 & 0.0000219 \\
\hline GO:0032612 interleukin-1 production & 39 & $14(35.9 \%)$ & 0.00000188 & 0.0000229 \\
\hline GO:0044093 positive regulation of molecular function & 982 & $125(12.7 \%)$ & 0.00000238 & 0.0000282 \\
\hline GO:0001666 response to hypoxia & 203 & $38(18.7 \%)$ & 0.00000278 & 0.0000323 \\
\hline GO:0044092 negative regulation of molecular function & 554 & $79(14.3 \%)$ & 0.00000316 & 0.0000359 \\
\hline $\begin{array}{l}\text { GO:0044087 regulation of cellular component } \\
\text { biogenesis }\end{array}$ & 240 & $42(17.5 \%)$ & 0.00000514 & 0.0000572 \\
\hline GO:0006793 phosphorus metabolic process & 1306 & $156(11.9 \%)$ & 0.00000559 & 0.0000609 \\
\hline GO:0042633 hair cycle & 73 & $19(26.0 \%)$ & 0.00000737 & 0.0000787 \\
\hline GO:0006897 endocytosis & 303 & $49(16.2 \%)$ & 0.00000861 & 0.0000883 \\
\hline GO:0010324 membrane invagination & 303 & 49 (16.2\%) & 0.00000861 & 0.0000883 \\
\hline GO:0022404 molting cycle process & 69 & $18(26.1 \%)$ & 0.0000123 & 0.000124 \\
\hline GO:0007599 hemostasis & 512 & $72(14.1 \%)$ & 0.0000139 & 0.000137 \\
\hline GO:0006952 defense response & 984 & $121(12.3 \%)$ & 0.000018 & 0.000175 \\
\hline GO:0035239 tube morphogenesis & 220 & $38(17.3 \%)$ & 0.0000193 & 0.000183 \\
\hline GO:0007596 blood coagulation & 508 & $71(14.0 \%)$ & 0.0000196 & 0.000183 \\
\hline GO:0016337 cell-cell adhesion & 330 & $51(15.5 \%)$ & 0.0000205 & 0.000188 \\
\hline GO:0006066 alcohol metabolic process & 521 & $72(13.8 \%)$ & 0.0000251 & 0.000226 \\
\hline GO:0031589 cell-substrate adhesion & 169 & $31(18.3 \%)$ & 0.0000336 & 0.000297 \\
\hline GO:0061061 muscle structure development & 363 & $54(14.9 \%)$ & 0.0000352 & 0.000307 \\
\hline GO:0090132 epithelium migration & 18 & $8(44.4 \%)$ & 0.0000534 & 0.000451 \\
\hline GO:0032844 regulation of homeostatic process & 181 & $32(17.7 \%)$ & 0.0000535 & 0.000451 \\
\hline GO:0048608 reproductive structure development & 224 & $37(16.5 \%)$ & 0.0000663 & 0.000544 \\
\hline GO:0009725 response to hormone stimulus & 660 & $85(12.9 \%)$ & 0.0000665 & 0.000544 \\
\hline GO:0043412 macromolecule modification & 2212 & $236(10.7 \%)$ & 0.0000779 & 0.000618 \\
\hline GO:0042493 response to drug & 338 & $50(14.8 \%)$ & 0.000078 & 0.000618 \\
\hline GO:0022607 cellular component assembly & 1282 & $146(11.4 \%)$ & 0.000118 & 0.000918 \\
\hline $\begin{array}{l}\text { GO:0060560 developmental growth involved in } \\
\text { morphogenesis }\end{array}$ & 97 & $20(20.6 \%)$ & 0.000158 & 0.00122 \\
\hline $\begin{array}{l}\text { GO:0071706 tumor necrosis factor superfamily } \\
\text { cytokine production }\end{array}$ & 62 & $15(24.2 \%)$ & 0.000162 & 0.00122 \\
\hline GO:0010876 lipid localization & 200 & $33(16.5 \%)$ & 0.000164 & 0.00122 \\
\hline GO:0045087 innate immune response & 440 & $60(13.6 \%)$ & 0.000168 & 0.00124 \\
\hline $\begin{array}{l}\text { GO:0044259 multicellular organismal macromolecule } \\
\text { metabolic process }\end{array}$ & 50 & $13(26.0 \%)$ & 0.000203 & 0.00147 \\
\hline GO:0032943 mononuclear cell proliferation & 153 & $27(17.6 \%)$ & 0.000205 & 0.00147 \\
\hline GO:0042592 homeostatic process & 996 & $116(11.6 \%)$ & 0.00026 & 0.00184 \\
\hline GO:0006979 response to oxidative stress & 223 & $35(15.7 \%)$ & 0.000286 & 0.00197 \\
\hline GO:0007620 copulation & 22 & $8(36.4 \%)$ & 0.000286 & 0.00197 \\
\hline GO:0006887 exocytosis & 241 & $37(15.4 \%)$ & 0.000304 & 0.00207 \\
\hline GO:0060056 mammary gland involution & 13 & $6(46.2 \%)$ & 0.000377 & 0.00253 \\
\hline GO:0048839 inner ear development & 111 & $21(18.9 \%)$ & 0.000384 & 0.00254 \\
\hline GO:0061138 morphogenesis of a branching epithelium & 136 & $24(17.6 \%)$ & 0.00045 & 0.00294 \\
\hline GO:0001935 endothelial cell proliferation & 68 & $15(22.1 \%)$ & 0.000478 & 0.00308 \\
\hline GO:0040008 regulation of growth & 440 & $58(13.2 \%)$ & 0.000514 & 0.00328 \\
\hline GO:0019538 protein metabolic process & 3414 & $339(9.9 \%)$ & 0.000527 & 0.00332 \\
\hline
\end{tabular}




\begin{tabular}{|c|c|c|c|c|}
\hline GO:0032535 regulation of cellular component size & 146 & $25(17.1 \%)$ & 0.000552 & 0.00342 \\
\hline GO:0048598 embryonic morphogenesis & 385 & $52(13.5 \%)$ & 0.000556 & 0.00342 \\
\hline GO:0055082 cellular chemical homeostasis & 510 & $65(12.7 \%)$ & 0.000613 & 0.00373 \\
\hline GO:0031099 regeneration & 115 & $21(18.3 \%)$ & 0.000627 & 0.00377 \\
\hline GO:0007584 response to nutrient & 225 & $34(15.1 \%)$ & 0.000699 & 0.00415 \\
\hline GO:0003179 heart valve morphogenesis & 10 & $5(50.0 \%)$ & 0.000767 & 0.00442 \\
\hline GO:0071495 cellular response to endogenous stimulus & 381 & $51(13.4 \%)$ & 0.000768 & 0.00442 \\
\hline GO:0016486 peptide hormone processing & 25 & $8(32.0 \%)$ & 0.00077 & 0.00442 \\
\hline GO:0090066 regulation of anatomical structure size & 245 & $36(14.7 \%)$ & 0.000838 & 0.00476 \\
\hline GO:0030728 ovulation & 20 & $7(35.0 \%)$ & 0.000903 & 0.00508 \\
\hline GO:0009612 response to mechanical stimulus & 111 & $20(18.0 \%)$ & 0.000994 & 0.00553 \\
\hline GO:0031069 hair follicle morphogenesis & 26 & $8(30.8 \%)$ & 0.00103 & 0.00561 \\
\hline GO:0008038 neuron recognition & 26 & $8(30.8 \%)$ & 0.00103 & 0.00561 \\
\hline GO:0044282 small molecule catabolic process & 800 & $93(11.6 \%)$ & 0.0011 & 0.00593 \\
\hline GO:0048645 organ formation & 39 & $10(25.6 \%)$ & 0.00122 & 0.0065 \\
\hline GO:0003170 heart valve development & 11 & $5(45.5 \%)$ & 0.00131 & 0.00687 \\
\hline GO:0060349 bone morphogenesis & 46 & $11(23.9 \%)$ & 0.00131 & 0.00687 \\
\hline GO:0030595 leukocyte chemotaxis & 90 & $17(18.9 \%)$ & 0.00135 & 0.00695 \\
\hline GO:0071695 anatomical structure maturation & 27 & $8(29.6 \%)$ & 0.00136 & 0.00695 \\
\hline GO:0071496 cellular response to external stimulus & 190 & $29(15.3 \%)$ & 0.00142 & 0.00722 \\
\hline GO:0071702 organic substance transport & 498 & $62(12.4 \%)$ & 0.00145 & 0.0073 \\
\hline GO:0048546 digestive tract morphogenesis & 40 & $10(25.0 \%)$ & 0.0015 & 0.00748 \\
\hline GO:0051098 regulation of binding & 124 & $21(16.9 \%)$ & 0.0017 & 0.00835 \\
\hline GO:0009595 detection of biotic stimulus & 22 & $7(31.8 \%)$ & 0.00171 & 0.00835 \\
\hline $\begin{array}{l}\text { GO:0045137 development of primary sexual } \\
\text { characteristics }\end{array}$ & 203 & $30(14.8 \%)$ & 0.00199 & 0.00955 \\
\hline GO:0008104 protein localization & 1252 & $135(10.8 \%)$ & 0.00199 & 0.00955 \\
\hline GO:0070271 protein complex biogenesis & 604 & $72(11.9 \%)$ & 0.00203 & 0.00966 \\
\hline GO:0048286 lung alveolus development & 35 & $9(25.7 \%)$ & 0.00207 & 0.00972 \\
\hline GO:0009991 response to extracellular stimulus & 351 & $46(13.1 \%)$ & 0.00208 & 0.00972 \\
\hline GO:0046849 bone remodeling & 42 & $10(23.8 \%)$ & 0.00223 & 0.0103 \\
\hline GO:0021675 nerve development & 49 & $11(22.4 \%)$ & 0.00227 & 0.0104 \\
\hline $\begin{array}{l}\text { GO:0002568 somatic diversification of T cell receptor } \\
\text { genes }\end{array}$ & 4 & $3(75.0 \%)$ & 0.00229 & 0.0104 \\
\hline GO:0003012 muscle system process & 252 & $35(13.9 \%)$ & 0.00261 & 0.0118 \\
\hline GO:0001946 lymphangiogenesis & 8 & $4(50.0 \%)$ & 0.00274 & 0.0122 \\
\hline GO:0070997 neuron death & 146 & $23(15.8 \%)$ & 0.0028 & 0.0124 \\
\hline GO:0016042 lipid catabolic process & 226 & $32(14.2 \%)$ & 0.00287 & 0.0126 \\
\hline $\begin{array}{l}\text { GO:0045104 intermediate filament cytoskeleton } \\
\text { organization }\end{array}$ & 24 & $7(29.2 \%)$ & 0.00298 & 0.013 \\
\hline GO:0032602 chemokine production & 37 & $9(24.3 \%)$ & 0.00313 & 0.0135 \\
\hline GO:0001945 lymph vessel development & 13 & $5(38.5 \%)$ & 0.00316 & 0.0135 \\
\hline GO:0045165 cell fate commitment & 174 & $26(14.9 \%)$ & 0.00328 & 0.0139 \\
\hline GO:0003002 regionalization & 247 & $34(13.8 \%)$ & 0.00344 & 0.0145 \\
\hline $\begin{array}{l}\text { GO:0009792 embryo development ending in birth or } \\
\text { egg hatching }\end{array}$ & 399 & $50(12.5 \%)$ & 0.00348 & 0.0146 \\
\hline
\end{tabular}




\begin{tabular}{|c|c|c|c|c|c|}
\hline GO:0002089 & lens morphogenesis in camera-type eye & 19 & $6(31.6 \%)$ & 0.00382 & 0.0159 \\
\hline GO:0048754 & branching morphogenesis of a tube & 124 & $20(16.1 \%)$ & 0.00388 & 0.016 \\
\hline GO:0003013 & circulatory system process & 278 & $37(13.3 \%)$ & 0.00417 & 0.017 \\
\hline GO:0044248 & cellular catabolic process & 1483 & $154(10.4 \%)$ & 0.00436 & 0.0177 \\
\hline GO:0002088 & lens development in camera-type eye & 46 & $10(21.7 \%)$ & 0.00453 & 0.0181 \\
\hline GO:0042403 & thyroid hormone metabolic process & 14 & $5(35.7 \%)$ & 0.00457 & 0.0181 \\
\hline GO:0009415 & response to water & 9 & $4(44.4 \%)$ & 0.0046 & 0.0181 \\
\hline GO:0042640 & anagen & 9 & $4(44.4 \%)$ & 0.0046 & 0.0181 \\
\hline GO:0006082 & organic acid metabolic process & 799 & $89(11.1 \%)$ & 0.00472 & 0.0184 \\
\hline GO:0010817 & regulation of hormone levels & 357 & $45(12.6 \%)$ & 0.00483 & 0.0187 \\
\hline GO:0021536 & diencephalon development & 54 & $11(20.4 \%)$ & 0.00504 & 0.0194 \\
\hline GO:0030900 & forebrain development & 226 & $31(13.7 \%)$ & 0.00533 & 0.0202 \\
\hline GO:0048659 & smooth muscle cell proliferation & 62 & $12(19.4 \%)$ & 0.00537 & 0.0202 \\
\hline GO:0016197 & endosome transport & 119 & $19(16.0 \%)$ & 0.00538 & 0.0202 \\
\hline GO:0060512 & prostate gland morphogenesis & 27 & $7(25.9 \%)$ & 0.00611 & 0.0228 \\
\hline GO:0060343 & trabecula formation & 15 & $5(33.3 \%)$ & 0.00638 & 0.0237 \\
\hline GO:0010259 & multicellular organismal aging & 21 & $6(28.6 \%)$ & 0.0066 & 0.0243 \\
\hline GO:0007548 & sex differentiation & 230 & $31(13.5 \%)$ & 0.00687 & 0.0251 \\
\hline GO:0060008 & Sertoli cell differentiation & 10 & $4(40.0 \%)$ & 0.00716 & 0.0251 \\
\hline GO:0021984 & adenohypophysis development & 10 & $4(40.0 \%)$ & 0.00716 & 0.0251 \\
\hline GO:0001550 & ovarian cumulus expansion & 2 & $2(100.0 \%)$ & 0.0072 & 0.0251 \\
\hline GO:0002238 & response to molecule of fungal origin & 2 & $2(100.0 \%)$ & 0.0072 & 0.0251 \\
\hline GO:0006788 & heme oxidation & 2 & $2(100.0 \%)$ & 0.0072 & 0.0251 \\
\hline $\begin{array}{l}\text { GO:0045229 } \\
\text { organization }\end{array}$ & external encapsulating structure & 2 & $2(100.0 \%)$ & 0.0072 & 0.0251 \\
\hline GO:0042335 & cuticle development & 2 & $2(100.0 \%)$ & 0.0072 & 0.0251 \\
\hline GO:0060711 & labyrinthine layer development & 28 & $7(25.0 \%)$ & 0.00756 & 0.0262 \\
\hline $\begin{array}{l}\text { GO:0072593 } \\
\text { process }\end{array}$ & reactive oxygen species metabolic & 98 & $16(16.3 \%)$ & 0.00818 & 0.0281 \\
\hline GO:0072006 & nephron development & 50 & $10(20.0 \%)$ & 0.00838 & 0.0287 \\
\hline GO:0061383 & trabecula morphogenesis & 16 & $5(31.2 \%)$ & 0.00864 & 0.0292 \\
\hline GO:0006805 & xenobiotic metabolic process & 142 & $21(14.8 \%)$ & 0.00865 & 0.0292 \\
\hline GO:0048588 & developmental cell growth & 74 & $13(17.6 \%)$ & 0.00891 & 0.0299 \\
\hline GO:0035019 & somatic stem cell maintenance & 29 & $7(24.1 \%)$ & 0.00926 & 0.0308 \\
\hline GO:0035148 & tube formation & 83 & $14(16.9 \%)$ & 0.00973 & 0.0322 \\
\hline
\end{tabular}

Table S4 Enriched gene ontology level 3 categories of biological processes of under-expressed-TFs networks. Set size refers to the number of entities that have a UniProt ID as stated in the corresponding GO category at the ConsensusPathDB site. The number of candidates contained refers to amount of proteins identified in this study that appear as part of the GO category. P-values are calculated according to a hypergeometric test; $q$-values represent $p$-values corrected for multiple testing using the false discovery rate method. 


\begin{tabular}{|c|c|c|c|c|}
\hline Pathway name & $\begin{array}{l}\text { Set } \\
\text { size }\end{array}$ & $\begin{array}{l}\text { Candidates } \\
\text { contained }\end{array}$ & P-value & Q-value \\
\hline Fanconi anemia pathway & 47 & 32 & $4.66 \mathrm{E}-10$ & $6.80 \mathrm{E}-08$ \\
\hline AP-1 transcription factor network & 70 & 26 & $2.98 \mathrm{E}-08$ & $1.45 \mathrm{E}-06$ \\
\hline ATR signaling pathway & 37 & 26 & $2.98 \mathrm{E}-08$ & $1.45 \mathrm{E}-06$ \\
\hline E2F transcription factor network & 77 & 38 & $8.05 \mathrm{E}-08$ & $2.94 \mathrm{E}-06$ \\
\hline PLK1 signaling events & 44 & 24 & $1.19 \mathrm{E}-07$ & $3.48 \mathrm{E}-06$ \\
\hline Regulation of Telomerase & 73 & 23 & $2.38 \mathrm{E}-07$ & $5.80 \mathrm{E}-06$ \\
\hline Direct p53 effectors & 144 & 21 & $9.54 \mathrm{E}-07$ & $1.74 \mathrm{E}-05$ \\
\hline FOXM1 transcription factor network & 42 & 21 & $9.54 \mathrm{E}-07$ & $1.74 \mathrm{E}-05$ \\
\hline Beta1 integrin cell surface interactions & 66 & 20 & $1.91 \mathrm{E}-06$ & $3.09 \mathrm{E}-05$ \\
\hline Aurora B signaling & 43 & 19 & $3.81 \mathrm{E}-06$ & $5.06 \mathrm{E}-05$ \\
\hline Regulation of nuclear SMAD2/3 signaling & 78 & 19 & $3.81 \mathrm{E}-06$ & $5.06 \mathrm{E}-05$ \\
\hline C-MYB transcription factor network & 85 & 18 & $7.63 \mathrm{E}-06$ & $7.43 \mathrm{E}-05$ \\
\hline ATF-2 transcription factor network & 61 & 18 & $7.63 \mathrm{E}-06$ & $7.43 \mathrm{E}-05$ \\
\hline Regulation of retinoblastoma protein & 66 & 18 & 7.63E-06 & $7.43 \mathrm{E}-05$ \\
\hline PDGFR-beta signaling pathway & 130 & 18 & $7.63 \mathrm{E}-06$ & $7.43 \mathrm{E}-05$ \\
\hline Validated targets of C-MYC transcriptional activation & 87 & 26 & $8.80 \mathrm{E}-06$ & $8.03 \mathrm{E}-05$ \\
\hline HIF-1-alpha transcription factor network & 66 & 17 & $1.53 \mathrm{E}-05$ & 0.000117 \\
\hline BARD1 signaling events & 31 & 17 & $1.53 \mathrm{E}-05$ & 0.000117 \\
\hline p73 transcription factor network & 79 & 17 & $1.53 \mathrm{E}-05$ & 0.000117 \\
\hline Validated nuclear estrogen receptor alpha network & 66 & 16 & $3.05 \mathrm{E}-05$ & 0.000171 \\
\hline Caspase cascade in apoptosis & 53 & 16 & $3.05 \mathrm{E}-05$ & 0.000171 \\
\hline ATM pathway & 35 & 16 & $3.05 \mathrm{E}-05$ & 0.000171 \\
\hline Validated targets of C-MYC transcriptional repression & 75 & 16 & $3.05 \mathrm{E}-05$ & 0.000171 \\
\hline Coregulation of Androgen receptor activity & 66 & 16 & $3.05 E-05$ & 0.000171 \\
\hline Integrins in angiogenesis & 65 & 16 & $3.05 \mathrm{E}-05$ & 0.000171 \\
\hline Glucocorticoid receptor regulatory network & 80 & 16 & $3.05 \mathrm{E}-05$ & 0.000171 \\
\hline Signaling events mediated by HDAC Class III & 40 & 17 & 0.000117 & 0.000575 \\
\hline ErbB1 downstream signaling & 108 & 14 & 0.000122 & 0.000575 \\
\hline p53 pathway & 59 & 14 & 0.000122 & 0.000575 \\
\hline mTOR signaling pathway & 65 & 14 & 0.000122 & 0.000575 \\
\hline IL4-mediated signaling events & 66 & 14 & 0.000122 & 0.000575 \\
\hline Validated transcriptional targets of TAp63 isoforms & 55 & 13 & 0.000244 & 0.00105 \\
\hline Syndecan-1-mediated signaling events & 44 & 13 & 0.000244 & 0.00105 \\
\hline Regulation of Androgen receptor activity & 51 & 13 & 0.000244 & 0.00105 \\
\hline HIF-2-alpha transcription factor network & 36 & 12 & 0.000488 & 0.00183 \\
\hline $\begin{array}{l}\text { Validated transcriptional targets of AP1 family members } \\
\text { Fra1 and Fra2 }\end{array}$ & 37 & 12 & 0.000488 & 0.00183 \\
\hline RhoA signaling pathway & 47 & 12 & 0.000488 & 0.00183 \\
\hline Beta3 integrin cell surface interactions & 44 & 12 & 0.000488 & 0.00183 \\
\hline RAC1 signaling pathway & 54 & 12 & 0.000488 & 0.00183 \\
\hline
\end{tabular}




\begin{tabular}{|c|c|c|c|c|}
\hline Regulation of RhoA activity & 47 & 11 & 0.000977 & 0.00297 \\
\hline Notch-mediated HES/HEY network & 48 & 11 & 0.000977 & 0.00297 \\
\hline CXCR4-mediated signaling events & 89 & 11 & 0.000977 & 0.00297 \\
\hline Validated transcriptional targets of deltaNp63 isoforms & 47 & 11 & 0.000977 & 0.00297 \\
\hline Downstream signaling in naïve CD8+ T cells & 68 & 11 & 0.000977 & 0.00297 \\
\hline IL2-mediated signaling events & 56 & 11 & 0.000977 & 0.00297 \\
\hline FoxO family signaling & 50 & 11 & 0.000977 & 0.00297 \\
\hline FOXA2 and FOXA3 transcription factor networks & 46 & 11 & 0.000977 & 0.00297 \\
\hline Signaling events mediated by HDAC Class I & 57 & 11 & 0.000977 & 0.00297 \\
\hline Endothelins & 65 & 10 & 0.00195 & 0.00483 \\
\hline HIV-1 Nef: Negative effector of Fas and TNF-alpha & 35 & 10 & 0.00195 & 0.00483 \\
\hline IL8- and CXCR1-mediated signaling events & 28 & 10 & 0.00195 & 0.00483 \\
\hline Thromboxane $A 2$ receptor signaling & 57 & 10 & 0.00195 & 0.00483 \\
\hline Arf6 trafficking events & 50 & 10 & 0.00195 & 0.00483 \\
\hline $\begin{array}{l}\text { Role of Calcineurin-dependent NFAT signaling in } \\
\text { lymphocytes }\end{array}$ & 57 & 10 & 0.00195 & 0.00483 \\
\hline $\begin{array}{l}\text { Calcineurin-regulated NFAT-dependent transcription in } \\
\text { lymphocytes }\end{array}$ & 50 & 10 & 0.00195 & 0.00483 \\
\hline CDC42 signaling events & 72 & 10 & 0.00195 & 0.00483 \\
\hline Signaling events mediated by PTP1B & 53 & 10 & 0.00195 & 0.00483 \\
\hline $\begin{array}{l}\text { Signaling events mediated by Hepatocyte Growth Factor } \\
\text { Receptor (c-Met) }\end{array}$ & 84 & 10 & 0.00195 & 0.00483 \\
\hline LKB1 signaling events & 45 & 10 & 0.00195 & 0.00483 \\
\hline $\begin{array}{l}\text { Regulation of nuclear beta catenin signaling and target } \\
\text { gene transcription }\end{array}$ & 81 & 11 & 0.00384 & 0.00803 \\
\hline Regulation of RAC1 activity & 39 & 9 & 0.00391 & 0.00803 \\
\hline LPA receptor mediated events & 63 & 9 & 0.00391 & 0.00803 \\
\hline Signaling events mediated by PRL & 24 & 9 & 0.00391 & 0.00803 \\
\hline p75(NTR)-mediated signaling & 71 & 9 & 0.00391 & 0.00803 \\
\hline Ceramide signaling pathway & 48 & 9 & 0.00391 & 0.00803 \\
\hline IL8- and CXCR2-mediated signaling events & 34 & 9 & 0.00391 & 0.00803 \\
\hline Hedgehog signaling events mediated by Gli proteins & 49 & 9 & 0.00391 & 0.00803 \\
\hline FGF signaling pathway & 50 & 9 & 0.00391 & 0.00803 \\
\hline Neurotrophic factor-mediated Trk receptor signaling & 65 & 9 & 0.00391 & 0.00803 \\
\hline IL6-mediated signaling events & 48 & 9 & 0.00391 & 0.00803 \\
\hline Class I PI3K signaling events & 45 & 9 & 0.00391 & 0.00803 \\
\hline C-MYC pathway & 25 & 8 & 0.00781 & 0.0137 \\
\hline FAS (CD95) signaling pathway & 35 & 8 & 0.00781 & 0.0137 \\
\hline Aurora A signaling & 31 & 8 & 0.00781 & 0.0137 \\
\hline PAR1-mediated thrombin signaling events & 44 & 8 & 0.00781 & 0.0137 \\
\hline IL12-mediated signaling events & 64 & 8 & 0.00781 & 0.0137 \\
\hline $\begin{array}{l}\text { Signaling events mediated by Stem cell factor receptor (c- } \\
\text { Kit) }\end{array}$ & 53 & 8 & 0.00781 & 0.0137 \\
\hline
\end{tabular}




\begin{tabular}{|l|l|l|l|l|}
\hline EPO signaling pathway & 34 & 8 & 0.00781 & 0.0137 \\
\hline $\begin{array}{l}\text { RXR and RAR heterodimerization with other nuclear } \\
\text { receptor }\end{array}$ & 26 & 8 & 0.00781 & 0.0137 \\
\hline FOXA1 transcription factor network & 45 & 8 & 0.00781 & 0.0137 \\
\hline Insulin Pathway & 48 & 8 & 0.00781 & 0.0137 \\
\hline Syndecan-4-mediated signaling events & 32 & 8 & 0.00781 & 0.0137 \\
\hline Signaling events mediated by VEGFR1 and VEGFR2 & 72 & 8 & 0.00781 & 0.0137 \\
\hline
\end{tabular}

Table S5 Enriched PID pathway-based sets of over-expressed transcripts. Set size refers to the number of entities that have a Uniprot ID as stated in the corresponding PID pathway-based set at the ConsensusPathDB site. The number of candidates contained refers to amount of proteins which are part of the extended network and appear as part of the pathway. P-values are calculated according to a hypergeometric test; q-values represent p-values corrected for multiple testing using the false discovery rate method.

\begin{tabular}{|l|l|l|l|l|}
\hline Pathway name & Set size & $\begin{array}{l}\text { Candidates } \\
\text { contained }\end{array}$ & P-value & Q-value \\
\hline $\begin{array}{l}\text { Validated transcriptional targets of deltaNp63 } \\
\text { isoforms }\end{array}$ & 47 & $17(36.2 \%)$ & $9.63 \mathrm{E}-06$ & 0.00177 \\
\hline Validated transcriptional targets of TAp63 isoforms & 55 & $16(29.6 \%)$ & 0.000268 & 0.0247 \\
\hline $\begin{array}{l}\text { E-cadherin signaling in the nascent adherens } \\
\text { junction }\end{array}$ & 39 & $12(31.6 \%)$ & 0.000823 & 0.0406 \\
\hline ErbB receptor signaling network & 16 & $7(43.8 \%)$ & 0.0012 & 0.0406 \\
\hline a6b1 and a6b4 Integrin signaling & 46 & $13(28.9 \%)$ & 0.00129 & 0.0406 \\
\hline $\begin{array}{l}\text { Posttranslational regulation of adherens junction } \\
\text { stability and dissassembly }\end{array}$ & 57 & $15(26.8 \%)$ & 0.00132 & 0.0406 \\
\hline Endogenous TLR signaling & 26 & $9(34.6 \%)$ & 0.00179 & 0.0469 \\
\hline Direct p53 effectors & 144 & $28(19.9 \%)$ & 0.00277 & 0.0637 \\
\hline $\begin{array}{l}\text { Calcineurin-regulated NFAT-dependent transcription } \\
\text { in lymphocytes }\end{array}$ & 50 & $13(26.0 \%)$ & 0.00362 & 0.0739 \\
\hline Nectin adhesion pathway & 32 & $9(29.0 \%)$ & 0.00678 & 0.113 \\
\hline Syndecan-2-mediated signaling events & 42 & $11(26.2 \%)$ & 0.00678 & 0.113 \\
\hline $\begin{array}{l}\text { Validated transcriptional targets of AP1 family } \\
\text { members Fra1 and Fra2 }\end{array}$ & 37 & $10(27.0 \%)$ & 0.00764 & 0.117 \\
\hline $\begin{array}{l}\text { Regulation of nuclear beta catenin signaling and } \\
\text { target gene transcription }\end{array}$ & 81 & $17(21.2 \%)$ & 0.00894 & 0.118 \\
\hline $\begin{array}{l}\text { Beta5 beta6 beta7 and beta8 integrin cell surface } \\
\text { interactions }\end{array}$ & 17 & $6(35.3 \%)$ & 0.00944 & 0.118 \\
\hline Cellular roles of Anthrax toxin & 22 & $7(31.8 \%)$ & 0.00964 & 0.118 \\
\hline
\end{tabular}

Table S6 Enriched PID pathway-based sets of sub-expressed transcripts. Set size refers to the number of entities that have a Uniprot ID as stated in the corresponding PID pathway-based set at the ConsensusPathDB site. The number of candidates contained refers to amount of proteins which are part of the extended network and appear as part of the pathway. P-values are calculated according to a hypergeometric test; $q$-values represent $p$-values corrected for multiple testing using the false discovery rate method. 


\begin{tabular}{|c|c|c|c|}
\hline $\begin{array}{l}\text { Over-expressed } \\
\text { transcripts }\end{array}$ & Folds ( $(\log 2)$ & $\begin{array}{l}\text { Under-expressed } \\
\text { transcripts }\end{array}$ & Folds ( $\log 2)$ \\
\hline AIFM2_HUMAN & 3.20816 & COIA1_HUMAN & -6.30929 \\
\hline KAT2A_HUMAN & 2.12567 & S10A2_HUMAN & -7.42878 \\
\hline MSH2_HUMAN & 2.86183 & VDR_HUMAN & -1.50616 \\
\hline LIF_HUMAN & 4.00723 & PML_HUMAN & -0.824712 \\
\hline DUS5_HUMAN & 1.86765 & MMP2_HUMAN & -8.4294 \\
\hline BBC3_HUMAN & 1.36481 & RIR2B_HUMAN & -1.16613 \\
\hline JMY_HUMAN & 1.20239 & PLK3_HUMAN & -0.634628 \\
\hline PCNA_HUMAN & 1.92967 & P63_HUMAN & -9.87507 \\
\hline SP1_HUMAN & 1.61246 & CSPG2_HUMAN & -4.44 \\
\hline BCL2_HUMAN & 3.49745 & SNAI2_HUMAN & -3.76971 \\
\hline TSC2_HUMAN & 0.85307 & SPB5_HUMAN & -2.50339 \\
\hline FOXA1_HUMAN & 4.50315 & ASC_HUMAN & -8.65988 \\
\hline GDF15_HUMAN & 2.69952 & Z385A_HUMAN & -1.76185 \\
\hline EDN2_HUMAN & 3.10815 & 1433S_HUMAN & -4.25544 \\
\hline DDB2_HUMAN & 2.26436 & PERP_HUMAN & -3.34831 \\
\hline XPO2_HUMAN & 1.21701 & DKK1_HUMAN & -3.13909 \\
\hline E2F1_HUMAN & 5.13942 & MDM2_HUMAN & -1.01875 \\
\hline ASPP2_HUMAN & 0.965443 & CD82_HUMAN & -4.86454 \\
\hline APAF_HUMAN & 1.06935 & R144B_HUMAN & -3.6513 \\
\hline IBP3_HUMAN & 6.04069 & BTG2_HUMAN & -4.20738 \\
\hline \multirow[t]{8}{*}{ E2F2_HUMAN } & 6.42348 & BKRB2_HUMAN & -2.06574 \\
\hline & & TIGAR_HUMAN & -2.66362 \\
\hline & & GA45A_HUMAN & -3.12129 \\
\hline & & TGFA_HUMAN & -2.43847 \\
\hline & & CAV1_HUMAN & -5.35133 \\
\hline & & BAK_HUMAN & -2.04026 \\
\hline & & IRF5_HUMAN & -3.13589 \\
\hline & & T53I1_HUMAN & -6.09755 \\
\hline
\end{tabular}

Table S7: list of over and under expressed transcripts members of the regulatory network "Direct effectors of p53" 


\begin{tabular}{|l|l|l|l|l|}
\hline pathway name & set size & $\begin{array}{l}\text { candidates } \\
\text { contained }\end{array}$ & p-value & q-value \\
\hline Steroid biosynthesis & 17 & $10(58.8 \%)$ & $3.71 \mathrm{E}-06$ & 0.000775 \\
\hline Tight junction & 132 & $33(25.0 \%)$ & $1.17 \mathrm{E}-05$ & 0.00122 \\
\hline Endocytosis & 202 & $44(21.8 \%)$ & $2.07 \mathrm{E}-05$ & 0.00144 \\
\hline Adherens junction & 73 & $20(27.4 \%)$ & 0.00016 & 0.00836 \\
\hline alpha-Linolenic acid metabolism & 21 & $9(42.9 \%)$ & 0.000287 & 0.0102 \\
\hline Axon guidance & 129 & $29(22.5 \%)$ & 0.000292 & 0.0102 \\
\hline Cell adhesion molecules (CAMs) & 133 & $29(21.8 \%)$ & 0.000502 & 0.0143 \\
\hline Arachidonic acid metabolism & 63 & $17(27.0 \%)$ & 0.00059 & 0.0143 \\
\hline Salmonella infection & 86 & $21(24.4 \%)$ & 0.000618 & 0.0143 \\
\hline VEGF signaling pathway & 76 & $19(25.0 \%)$ & 0.000811 & 0.0161 \\
\hline Amoebiasis & 108 & $24(22.6 \%)$ & 0.000847 & 0.0161 \\
\hline Fc gamma R-mediated phagocytosis & 97 & $22(23.2 \%)$ & 0.000999 & 0.0168 \\
\hline Pathogenic Escherichia coli infection & 55 & $15(27.3 \%)$ & 0.00108 & 0.0168 \\
\hline Regulation of actin cytoskeleton & 212 & $40(18.9 \%)$ & 0.00112 & 0.0168 \\
\hline Terpenoid backbone biosynthesis & 21 & $8(38.1 \%)$ & 0.00158 & 0.0221 \\
\hline Notch signaling pathway & 47 & $13(27.7 \%)$ & 0.00199 & 0.026 \\
\hline Bladder cancer & 42 & $12(28.6 \%)$ & 0.00217 & 0.0267 \\
\hline Phosphatidylinositol signaling system & 80 & $18(22.5 \%)$ & 0.00387 & 0.045 \\
\hline Linoleic acid metabolism & 29 & $9(31.0 \%)$ & 0.00416 & 0.0458 \\
\hline Focal adhesion & 200 & $36(18.0 \%)$ & 0.0044 & 0.0459 \\
\hline Bacterial invasion of epithelial cells & 70 & $16(22.9 \%)$ & 0.00534 & 0.0531 \\
\hline Glioma & 65 & $15(23.1 \%)$ & 0.00626 & 0.0586 \\
\hline Leukocyte transendothelial migration & 116 & $23(19.8 \%)$ & 0.00644 & 0.0586 \\
\hline Biosynthesis of unsaturated fatty acids & 21 & $7(33.3 \%)$ & 0.00728 & 0.0634 \\
\hline Fc epsilon RI signaling pathway & 81 & $17(21.5 \%)$ & 0.00787 & 0.0658 \\
\hline
\end{tabular}

Table S8 Enriched KEGG pathway-based sets of under-expressed transcripts. Set size refers to the number of entities that have a Uniprot ID as stated in the corresponding KEGG pathway-based set at the ConsensusPathDB site. The number of candidates contained refers to amount of proteins which are part of the extended network and appear as part of the pathway. P-values are calculated according to a hypergeometric test; q-values represent $p$-values corrected for multiple testing using the false discovery rate method. 


\begin{tabular}{|c|c|c|c|c|}
\hline Gene Ontology term & $\begin{array}{l}\text { Set } \\
\text { size }\end{array}$ & $\begin{array}{l}\text { Candidates } \\
\text { contained }\end{array}$ & P-value & Q-value \\
\hline GO:0043933 macromolecular complex subunit organization & 1176 & $68(5.8 \%)$ & $1.74 \mathrm{E}-21$ & $3.45 \mathrm{E}-19$ \\
\hline $\begin{array}{l}\text { GO:0071842 cellular component organization at cellular } \\
\text { level }\end{array}$ & 3203 & $116(3.6 \%)$ & $3.25 \mathrm{E}-20$ & $3.24 \mathrm{E}-18$ \\
\hline GO:0022607 cellular component assembly & 1520 & $72(4.7 \%)$ & 7.10E-18 & $4.71 \mathrm{E}-16$ \\
\hline GO:0007018 microtubule-based movement & 144 & $18(12.5 \%)$ & $1.27 \mathrm{E}-11$ & $6.31 \mathrm{E}-10$ \\
\hline GO:0019058 viral infectious cycle & 235 & $21(9.0 \%)$ & $1.43 \mathrm{E}-10$ & $5.71 \mathrm{E}-09$ \\
\hline GO:0046907 intracellular transport & 1101 & $45(4.1 \%)$ & $3.43 \mathrm{E}-09$ & $1.14 \mathrm{E}-07$ \\
\hline $\begin{array}{l}\text { GO:0072594 establishment of protein localization to } \\
\text { organelle }\end{array}$ & 206 & $18(8.8 \%)$ & 4.35E-09 & $1.24 \mathrm{E}-07$ \\
\hline GO:0035966 response to topologically incorrect protein & 139 & $15(10.8 \%)$ & $5.31 \mathrm{E}-09$ & $1.29 \mathrm{E}-07$ \\
\hline $\begin{array}{l}\text { GO:0045104 intermediate filament cytoskeleton } \\
\text { organization }\end{array}$ & 27 & $8(29.6 \%)$ & $5.86 \mathrm{E}-09$ & $1.29 \mathrm{E}-07$ \\
\hline GO:0022411 cellular component disassembly & 291 & $21(7.2 \%)$ & 7.09E-09 & $1.41 \mathrm{E}-07$ \\
\hline GO:0019080 viral genome expression & 155 & $15(9.7 \%)$ & $2.17 \mathrm{E}-08$ & $3.59 \mathrm{E}-07$ \\
\hline GO:0019083 viral transcription & 155 & $15(9.7 \%)$ & $2.17 \mathrm{E}-08$ & $3.59 \mathrm{E}-07$ \\
\hline GO:0015031 protein transport & 1216 & $44(3.6 \%)$ & $1.82 \mathrm{E}-07$ & $2.79 \mathrm{E}-06$ \\
\hline GO:0044260 cellular macromolecule metabolic process & 6676 & $144(2.2 \%)$ & $1.35 \mathrm{E}-06$ & $1.92 \mathrm{E}-05$ \\
\hline GO:0008104 protein localization & 1523 & $48(3.2 \%)$ & $2.59 \mathrm{E}-06$ & $3.44 \mathrm{E}-05$ \\
\hline GO:0070271 protein complex biogenesis & 755 & $30(4.0 \%)$ & $3.24 \mathrm{E}-06$ & 4.03E-05 \\
\hline GO:0019047 provirus integration & 8 & $4(50.0 \%)$ & $4.01 \mathrm{E}-06$ & $4.43 \mathrm{E}-05$ \\
\hline GO:0030069 lysogeny & 8 & $4(50.0 \%)$ & $4.01 \mathrm{E}-06$ & $4.43 \mathrm{E}-05$ \\
\hline GO:0070727 cellular macromolecule localization & 895 & $33(3.7 \%)$ & $4.92 \mathrm{E}-06$ & $5.15 \mathrm{E}-05$ \\
\hline GO:0071843 cellular component biogenesis at cellular level & 245 & $15(6.1 \%)$ & $8.33 \mathrm{E}-06$ & $8.29 \mathrm{E}-05$ \\
\hline GO:0019538 protein metabolic process & 3827 & $88(2.3 \%)$ & $6.38 \mathrm{E}-05$ & 0.000605 \\
\hline GO:0044248 cellular catabolic process & 1683 & $47(2.8 \%)$ & $7.48 \mathrm{E}-05$ & 0.000677 \\
\hline GO:0016052 carbohydrate catabolic process & 170 & $11(6.5 \%)$ & $8.20 \mathrm{E}-05$ & 0.000709 \\
\hline GO:0044282 small molecule catabolic process & 319 & $15(4.7 \%)$ & 0.000172 & 0.00142 \\
\hline GO:0048731 system development & 3281 & $75(2.3 \%)$ & 0.000347 & 0.00276 \\
\hline $\begin{array}{l}\text { GO:0006139 nucleobase-containing compound metabolic } \\
\text { process }\end{array}$ & 5195 & $108(2.1 \%)$ & 0.000437 & 0.00335 \\
\hline GO:0034641 cellular nitrogen compound metabolic process & 5621 & $115(2.0 \%)$ & 0.000478 & 0.00353 \\
\hline GO:0019059 initiation of viral infection & 25 & $4(16.0 \%)$ & 0.000586 & 0.00403 \\
\hline GO:0007596 blood coagulation & 518 & $19(3.7 \%)$ & 0.000588 & 0.00403 \\
\hline GO:0007599 hemostasis & 522 & $19(3.6 \%)$ & 0.000645 & 0.00428 \\
\hline GO:0009057 macromolecule catabolic process & 844 & $26(3.1 \%)$ & 0.000859 & 0.00552 \\
\hline GO:0016192 vesicle-mediated transport & 945 & $28(3.0 \%)$ & 0.00101 & 0.00625 \\
\hline GO:0000086 G2/M transition of mitotic cell cycle & 145 & $8(5.5 \%)$ & 0.00212 & 0.0128 \\
\hline GO:0022614 membrane to membrane docking & 5 & $2(40.0 \%)$ & 0.00239 & 0.0138 \\
\hline GO:0030154 cell differentiation & 2562 & $58(2.3 \%)$ & 0.00243 & 0.0138 \\
\hline GO:0030168 platelet activation & 222 & $10(4.5 \%)$ & 0.0028 & 0.0155 \\
\hline
\end{tabular}




\begin{tabular}{|c|c|c|c|c|c|c|c|c|c|c|c|c|}
\hline Swisprot ID & Rep_1 & $\begin{array}{l}\text { Mass_ } \\
1\end{array}$ & $\begin{array}{l}\text { Score_ } \\
1\end{array}$ & $\begin{array}{l}\text { Matches_ } \\
1\end{array}$ & Rep_2 & Mass_2 & $\begin{array}{l}\text { Score_ } \\
2\end{array}$ & $\begin{array}{l}\text { Matches_ } \\
2\end{array}$ & Rep_3 & $\begin{array}{l}\text { Mass_ } \\
3\end{array}$ & Score_3 & $\begin{array}{l}\text { Matches_ } \\
3\end{array}$ \\
\hline 1433B & & & & & & & & & 1433B & 28179 & 78 & $5(2)$ \\
\hline 1433E & & & & & $1433 E$ & 29326 & 47 & $2(1)$ & 1433E & 29326 & 128 & $3(3)$ \\
\hline $1433 \mathrm{~F}$ & & & & & $1433 \mathrm{~F}$ & 28372 & 49 & $2(2)$ & & & & \\
\hline 1433G & & & & & 1433G & 28456 & 47 & $3(1)$ & 1433G & 28456 & 78 & $4(2)$ \\
\hline 1433S & & & & & $1433 S$ & 27871 & 49 & $2(2)$ & & & & \\
\hline 14332 & & & & & 14332 & 27899 & 136 & $5(4)$ & $1433 Z$ & 27899 & 140 & $7(4)$ \\
\hline $4 F 2$ & & & & & & & & & $4 F 2$ & 68180 & 52 & $1(1)$ \\
\hline ACDSB & ACDSB & 47797 & 34 & $1(1)$ & & & & & & & & \\
\hline ACOXL & & & & & ACOXL & 62383 & 37 & $1(1)$ & ACOXL & 62383 & 44 & $2(1)$ \\
\hline ACTA & ACTA & 42381 & 37 & $1(1)$ & & & & & & & & \\
\hline ACTB & & & & & ACTB & 42052 & 76 & $3(2)$ & ACTB & 42052 & 401 & $13(11)$ \\
\hline ACTBL & & & & & ACTBL & 42318 & 63 & $4(1)$ & ACTBL & 42318 & 161 & $4(4)$ \\
\hline ACTBM & & & & & ACTBM & 42331 & 60 & $2(1)$ & & & & \\
\hline ACTC & & & & & & & & & ACTC & 42334 & 223 & $9(6)$ \\
\hline ACTN1 & & & & & & & & & ACTN1 & 103563 & 50 & $1(1)$ \\
\hline AHNK & & & & & AHNK & 629213 & 83 & $3(2)$ & & & & \\
\hline AHNK2 & & & & & & & & & AHNK2 & 617383 & 47 & $1(1)$ \\
\hline AINX & AINX & 55391 & 42 & $1(1)$ & & & & & & & & \\
\hline AKTS1 & & & & & & & & & AKTS1 & 27595 & 35 & $1(1)$ \\
\hline ALBU & & & & & & & & & ALBU & 71317 & 73 & $3(1)$ \\
\hline ALDOA & & & & & ALDOA & 39851 & 77 & $2(1)$ & ALDOA & 39851 & 187 & $7(5)$ \\
\hline \multicolumn{13}{|l|}{ ANO8 } \\
\hline ANXA2 & & & & & ANXA2 & 38808 & 54 & $1(1)$ & ANXA2 & 38808 & 108 & $4(4)$ \\
\hline ANXA5 & ANXA5 & 35971 & 59 & $2(1)$ & & & & & & & & \\
\hline AP3D1 & & & & & AP3D1 & 131159 & 36 & $1(1)$ & & & & \\
\hline ASAH1 & & & & & ASAH1 & 45087 & 33 & $1(1)$ & & & & \\
\hline ASAP2 & & & & & ASAP2 & 112835 & 47 & $1(1)$ & & & & \\
\hline ASSY & & & & & & & & & ASSY & 46786 & 44 & $1(1)$ \\
\hline ATR & & & & & ATR & 304764 & 38 & $2(1)$ & & & & \\
\hline
\end{tabular}




\begin{tabular}{|c|c|c|c|c|c|c|c|c|c|c|c|c|}
\hline HBB & & & & & $\mathrm{HBB}$ & 16102 & 46 & $2(1)$ & & & & \\
\hline HDGF & & & & & HDGF & 26886 & 68 & $4(3)$ & HDGF & 26886 & 75 & $4(2)$ \\
\hline HIP1R & & & & & & & & & HIP1R & 119999 & 36 & $1(1)$ \\
\hline HMGA1 & & & & & & & & & $\begin{array}{l}\text { HMGA } \\
1\end{array}$ & 11669 & 64 & $1(1)$ \\
\hline HMMR & & & & & HMMR & 84448 & 34 & $1(1)$ & & & & \\
\hline HNRCL & HNRCL & 32180 & 75 & 1(1) & & & & & & & & \\
\hline HNRPC & & & & & & & & & HNRPC & 33707 & 106 & $3(3)$ \\
\hline HNRPD & & & & & & & & & HNRPD & 38581 & 38 & $2(1)$ \\
\hline HNRPK & & & & & HNRPK & 51230 & 53 & $2(1)$ & HNRPK & 51230 & 133 & $4(4)$ \\
\hline HNRPU & & & & & HNRPU & 91269 & 116 & $6(5)$ & HNRPU & 91269 & 253 & $13(6)$ \\
\hline HPPD & & & & & & & & & HPPD & 45077 & 34 & $1(1)$ \\
\hline HS71L & HS71L & 70730 & 72 & $3(1)$ & & & & & & & & \\
\hline HS902 & & & & & HS902 & 39454 & 76 & $6(2)$ & & & & \\
\hline HS90A & & & & & HS90A & 85006 & 254 & $8(7)$ & HS90A & 85006 & 555 & $22(19)$ \\
\hline HS90B & & & & & HS90B & 83554 & 328 & $18(11)$ & HS90B & 83554 & 942 & $36(27)$ \\
\hline HSP71 & & & & & HSP71 & 70294 & 92 & $4(2)$ & HSP71 & 70294 & 240 & $12(9)$ \\
\hline HSP76 & & & & & HSP76 & 71440 & 40 & $1(1)$ & HSP76 & 71440 & 195 & $6(6)$ \\
\hline HSP7C & & & & & HSP7C & 71082 & 34 & $1(1)$ & HSP7C & 71082 & 330 & $13(10)$ \\
\hline HSPB1 & & & & & HSPB1 & 22826 & 59 & $2(2)$ & HSPB1 & 22826 & 79 & $3(2)$ \\
\hline HTSF1 & & & & & HTSF1 & 86371 & 39 & $1(1)$ & & & & \\
\hline HUWE1 & & & & & & & & & $\begin{array}{l}\text { HUWE } \\
1\end{array}$ & 485523 & 35 & $2(1)$ \\
\hline IF1AX & & & & & IF1AX & 16564 & 41 & $2(1)$ & & & & \\
\hline IF1AY & & & & & & & & & IF1AY & 16546 & 78 & $2(1)$ \\
\hline IF4A1 & & & & & IF4A1 & 46353 & 67 & $3(1)$ & IF4A1 & 46353 & 73 & $4(2)$ \\
\hline IF4B & & & & & & & & & IF4B & 69167 & 96 & $3(1)$ \\
\hline ILF2 & & & & & & & & & ILF2 & 43263 & 80 & $2(2)$ \\
\hline JKIP1 & & & & & & & & & JKIP1 & 73506 & 37 & $1(1)$ \\
\hline K1C10 & & & & & $\mathrm{K} 1 \mathrm{C} 10$ & 59020 & 111 & $5(3)$ & K1C10 & 59020 & 399 & $16(12)$ \\
\hline K1C12 & & & & & K1C12 & 53592 & 39 & $2(1)$ & & & & \\
\hline
\end{tabular}




\begin{tabular}{|c|c|c|c|c|c|c|c|c|c|c|c|c|}
\hline K1C13 & & & & & & & & & K1C13 & 49900 & 64 & $2(1)$ \\
\hline K1C14 & & & & & K1C14 & 51872 & 88 & $3(2)$ & K1C14 & 51872 & 177 & $6(6)$ \\
\hline K1C15 & K1C15 & 49409 & 53 & $3(2)$ & & & & & & & & \\
\hline K1C16 & & & & & & & & & K1C16 & 51578 & 187 & $7(7)$ \\
\hline K1C17 & & & & & K1C17 & 48361 & 35 & $3(1)$ & K1C17 & 48361 & 103 & $5(3)$ \\
\hline K1C18 & K1C18 & 48029 & 80 & $3(2)$ & & & & & & & & \\
\hline K1C19 & K1C19 & 44079 & 89 & $4(3)$ & & & & & & & & \\
\hline K1C25 & & & & & K1C25 & 49858 & 59 & $2(1)$ & & & & \\
\hline K1C27 & & & & & K1C27 & 50419 & 72 & $2(1)$ & & & & \\
\hline K1C28 & K1C28 & 51163 & 42 & $3(1)$ & & & & & & & & \\
\hline K1C9 & & & & & K1C9 & 62255 & 203 & $7(4)$ & K1C9 & 62255 & 60 & $2(1)$ \\
\hline $\mathrm{K} 1 \mathrm{H} 1$ & & & & & $\mathrm{~K} 1 \mathrm{H} 1$ & 48633 & 45 & $2(1)$ & & & & \\
\hline $\mathrm{K} 22 \mathrm{E}$ & & & & & K22E & 65678 & 87 & $5(2)$ & $\mathrm{K} 22 \mathrm{E}$ & 65678 & 104 & $7(3)$ \\
\hline K22O & & & & & $\mathrm{K} 22 \mathrm{O}$ & 66370 & 44 & $4(1)$ & $\mathrm{K} 22 \mathrm{O}$ & 66370 & 94 & $4(4)$ \\
\hline $\mathrm{K} 2 \mathrm{C} 1$ & & & & & $\mathrm{~K} 2 \mathrm{C} 1$ & 66170 & 350 & $13(11)$ & $\mathrm{K} 2 \mathrm{C} 1$ & 66170 & 282 & 11(9) \\
\hline K2C1B & & & & & $\mathrm{K} 2 \mathrm{C} 1 \mathrm{~B}$ & 62149 & 40 & $2(1)$ & & & & \\
\hline K2C4 & & & & & K2C4 & 57649 & 93 & $3(2)$ & K2C4 & 57649 & 65 & $2(1)$ \\
\hline K2C5 & & & & & $\mathrm{K} 2 \mathrm{C5}$ & 62568 & 33 & $1(1)$ & $\mathrm{K} 2 \mathrm{C} 5$ & 62568 & 175 & $10(8)$ \\
\hline K2C6A & & & & & K2C6A & 60293 & 44 & $3(1)$ & & & & \\
\hline K2C6B & & & & & $\mathrm{K} 2 \mathrm{C} 6 \mathrm{~B}$ & 60315 & 67 & $3(2)$ & K2C6B & 60315 & 88 & $4(2)$ \\
\hline $\mathrm{K} 2 \mathrm{C} 6 \mathrm{C}$ & & & & & & & & & $\mathrm{K} 2 \mathrm{C} 6 \mathrm{C}$ & 60273 & 253 & $15(11)$ \\
\hline $\mathrm{K} 2 \mathrm{C7}$ & & & & & $\mathrm{K} 2 \mathrm{C} 7$ & 51411 & 66 & $2(1)$ & & & & \\
\hline $\mathrm{K} 2 \mathrm{C} 73$ & & & & & $\mathrm{~K} 2 \mathrm{C} 73$ & 59457 & 36 & $2(1)$ & & & & \\
\hline K2C75 & & & & & K2C75 & 59809 & 57 & $3(1)$ & & & & \\
\hline K2C79 & & & & & K2C79 & 58085 & 60 & $2(1)$ & K2C79 & 58085 & 118 & $6(5)$ \\
\hline K2C8 & & & & & $\mathrm{K} 2 \mathrm{C} 8$ & 53671 & 86 & $3(1)$ & $\mathrm{K} 2 \mathrm{C} 8$ & 53671 & 63 & $4(2)$ \\
\hline K2C8O & $\mathrm{K} 2 \mathrm{C} 80$ & 51007 & 78 & $2(1)$ & & & & & & & & \\
\hline KCNG3 & & & & & KCNG3 & 50359 & 34 & $1(1)$ & & & & \\
\hline $\mathrm{KI} 20 \mathrm{~B}$ & & & & & KI2OB & 211868 & 36 & $1(1)$ & & & & \\
\hline KIF1A & & & & & KIF1A & 192541 & 36 & $2(1)$ & & & & \\
\hline KPYM & & & & & & & & & KPYM & 58470 & 61 & $1(1)$ \\
\hline
\end{tabular}




\begin{tabular}{|c|c|c|c|c|c|c|c|c|c|c|c|c|}
\hline $\mathrm{BCL7B}$ & BCL7B & 22195 & 37 & $1(1)$ & & & & & & & & \\
\hline BFSP1 & & & & & BFSP1 & 74784 & 44 & $1(1)$ & & & & \\
\hline BLK & & & & & & & & & BLK & 58126 & 51 & $1(1)$ \\
\hline BZW1 & & & & & & & & & BZW1 & 48184 & 44 & $2(1)$ \\
\hline BZW2 & & & & & BZW2 & 48360 & 47 & $1(1)$ & BZW2 & 48360 & 54 & $2(2)$ \\
\hline C1QBP & C1QBP & 31742 & 92 & $2(2)$ & & & & & & & & \\
\hline CALR & CALR & & & & & & & & CALR & 48283 & 35 & $1(1)$ \\
\hline CALU & CALU & 37198 & 36 & $3(1)$ & & & & & & & & \\
\hline CALX & & & & & & & & & CALX & 67982 & 49 & $1(1)$ \\
\hline CAPS2 & & & & & CAPS2 & 148895 & 34 & $1(1)$ & & & & \\
\hline CC110 & CC110 & 96726 & 49 & $2(1)$ & & & & & & & & \\
\hline CDK1 & & & & & & & & & CDK1 & 34131 & 51 & 1(1) \\
\hline \multicolumn{13}{|l|}{$\mathrm{CH} 60$} \\
\hline $\mathrm{CH} 60$ & & & & & $\mathrm{CH} 60$ & 61187 & 103 & $3(3)$ & $\mathrm{CH} 60$ & 61187 & 173 & $9(6)$ \\
\hline $\mathrm{CHM} 2 \mathrm{~A}$ & & & & & & & & & $\mathrm{CHM} 2 \mathrm{~A}$ & 25088 & 37 & $1(1)$ \\
\hline CHMP5 & & & & & & & & & CHMP5 & 24612 & 35 & $1(1)$ \\
\hline CHRC1 & & & & & & & & & CHRC1 & 14758 & 36 & $1(1)$ \\
\hline CLIC1 & & & & & & & & & CLIC1 & 27248 & 41 & 1(1) \\
\hline CNTLN & & & & & & & & & CNTLN & 162131 & 37 & 1(1) \\
\hline CNTP4 & & & & & CNTP4 & 147178 & 37 & $1(1)$ & CNTP4 & 147178 & 37 & 1(1) \\
\hline CO039 & CO039 & 112086 & 35 & $2(1)$ & & & & & & & & \\
\hline C06A1 & C06A1 & 108529 & 39 & $1(1)$ & & & & & & & & \\
\hline CTNA1 & & & & & & & & & CTNA1 & 100693 & 38 & 1(1) \\
\hline DDX21 & & & & & & & & & DDX21 & 87804 & 88 & $4(3)$ \\
\hline DIRA2 & DIRA2 & 22813 & 52 & $1(1)$ & & & & & & & & \\
\hline DNJA2 & DNJA2 & 46344 & 47 & $1(1)$ & & & & & & & & \\
\hline DPF3 & DPF3 & 44254 & 37 & $1(1)$ & & & & & & & & \\
\hline DX39A & DX39A & 49611 & 54 & $4(2)$ & & & & & & & & \\
\hline DYH2 & DYH2 & 510796 & 39 & $1(1)$ & & & & & & & & \\
\hline EBP2 & & & & & & & & & EBP2 & 34887 & 36 & $1(1)$ \\
\hline
\end{tabular}




\begin{tabular}{|c|c|c|c|c|c|c|c|c|c|c|c|c|}
\hline EEPD1 & EEPD1 & 62820 & 43 & $1(1)$ & & & & & & & & \\
\hline EF1A1 & & & & & EF1A1 & 50451 & 59 & $1(1)$ & EF1A1 & 50451 & 138 & $7(4)$ \\
\hline EF1B & & & & & EF1B & 24919 & 106 & $3(3)$ & EF1B & 24919 & 139 & $2(2)$ \\
\hline EF1D & & & & & EF1D & 31217 & 39 & $1(1)$ & EF1D & 31217 & 283 & $6(6)$ \\
\hline EFTU & & & & & EFTU & 49852 & 34 & $1(1)$ & EFTU & 49852 & 44 & $4(1)$ \\
\hline EIF3B & & & & & EIF3B & 92823 & 59 & $1(1)$ & EIF3B & 92823 & 224 & $10(8)$ \\
\hline EIF3J & & & & & EIF3J & 29159 & 60 & $1(1)$ & EIF3J & 29159 & 45 & $2(1)$ \\
\hline ENOA & ENOA & 47481 & 41 & $1(1)$ & ENOA & 47481 & 66 & $2(1)$ & ENOA & 47481 & 135 & $7(6)$ \\
\hline ENPL & ENPL & 92696 & 71 & $7(2)$ & & & & & & & & \\
\hline EZRI & & & & & & & & & EZRI & 69484 & 50 & $4(2)$ \\
\hline F10A1 & & & & & F10A1 & 41477 & 65 & $3(2)$ & F10A1 & 41477 & 200 & $8(4)$ \\
\hline F16A1 & & & & & & & & & F16A1 & 117744 & 36 & $2(1)$ \\
\hline G3BP1 & & & & & G3BP1 & 52189 & 49 & $4(3)$ & G3BP1 & 52189 & 326 & $11(10)$ \\
\hline G3BP2 & & & & & G3BP2 & 54145 & 47 & $3(2)$ & G3BP2 & 54145 & 60 & $3(2)$ \\
\hline G3P & & & & & G3P & 36201 & 64 & $3(2)$ & G3P & 36201 & 223 & $5(3)$ \\
\hline G3PT & G3PT & 44815 & 62 & $2(2)$ & & & & & & & & \\
\hline GBF1 & & & & & GBF1 & 208367 & 45 & $2(1)$ & & & & \\
\hline GBRA5 & GBRA5 & 52398 & 33 & $1(0)$ & & & & & & & & \\
\hline GFAP & & & & & GFAP & 49907 & 56 & $2(1)$ & & & & \\
\hline GIT2 & GIT2 & 85117 & 51 & $6(3)$ & & & & & & & & \\
\hline GLU2B & & & & & GLU2B & 60357 & 56 & $1(1)$ & GLU2B & 60357 & 71 & $3(1)$ \\
\hline GOGA1 & GOGA1 & 88244 & 42 & $1(1)$ & & & & & & & & \\
\hline GP128 & & & & & GP128 & 89992 & 35 & $1(1)$ & & & & \\
\hline GRP75 & & & & & & & & & GRP75 & 73920 & 46 & $1(1)$ \\
\hline GRP78 & & & & & GRP78 & 72402 & 142 & $7(4)$ & GRP78 & 72402 & 235 & $10(10)$ \\
\hline H33 & & & & & & & & & H33 & 15376 & 108 & $11(5)$ \\
\hline $\mathrm{H} 3 \mathrm{C}$ & & & & & $\mathrm{H} 3 \mathrm{C}$ & 15318 & 45 & $4(2)$ & $\mathrm{H} 3 \mathrm{C}$ & 15318 & 47 & $2(1)$ \\
\hline $\mathrm{H} 4$ & $\mathrm{H} 4$ & 11367 & 45 & $2(1)$ & & & & & & & & \\
\hline H90B2 & & & & & H90B2 & 44492 & 86 & $4(3)$ & H90B2 & 44492 & 245 & $12(8)$ \\
\hline H90B3 & & & & & & & & & H90B3 & 68624 & 346 & 14(11) \\
\hline H90B4 & & & & & H90B4 & 58855 & 64 & $5(3)$ & H90B4 & 58855 & 45 & $3(1)$ \\
\hline
\end{tabular}




\begin{tabular}{|c|c|c|c|c|c|c|c|c|c|c|c|c|}
\hline KRT35 & & & & & KRT35 & 51640 & 45 & $3(1)$ & & & & \\
\hline KRT38 & KRT38 & 52044 & 56 & $2(1)$ & & & & & & & & \\
\hline KT222 & KT222 & 34158 & 68 & $2(1)$ & & & & & & & & \\
\hline LA & & & & & & & & & LA & 46979 & 51 & $1(1)$ \\
\hline LC7L2 & & & & & & & & & LC7L2 & 46942 & 48 & $1(1)$ \\
\hline LDH6A & & & & & & & & & LDH6A & 36826 & 91 & $2(1)$ \\
\hline LDHA & & & & & & & & & LDHA & 36950 & 110 & $5(3)$ \\
\hline LDHB & & & & & & & & & LDHB & 36900 & 125 & $5(5)$ \\
\hline LEG3 & & & & & & & & & LEG3 & 26152 & 39 & $1(1)$ \\
\hline LIMA1 & & & & & LIMA1 & 85630 & 52 & $3(1)$ & LIMA1 & 85630 & 47 & $2(1)$ \\
\hline LMNA & & & & & LMNA & 74380 & 57 & $1(1)$ & LMNA & 74380 & 215 & $7(6)$ \\
\hline LMNB1 & & & & & & & & & LMNB1 & 66653 & 39 & $3(2)$ \\
\hline LMNB2 & & & & & & & & & LMNB2 & 67762 & 39 & $2(2)$ \\
\hline MAGD4 & MAGD4 & 81555 & 43 & $1(1)$ & & & & & & & & \\
\hline MATR3 & & & & & MATR3 & 95078 & 78 & $5(3)$ & MATR3 & 95078 & 38 & $4(1)$ \\
\hline MCM2 & & & & & & & & & MCM2 & 102516 & 40 & $1(1)$ \\
\hline MCM3 & & & & & MCM3 & 91551 & 46 & $1(1)$ & MCM3 & 91551 & 117 & $4(3)$ \\
\hline MDHM & MDHM & 35937 & 35 & $1(1)$ & & & & & & & & \\
\hline MIF & & & & & & & & & MIF & 12639 & 54 & $1(1)$ \\
\hline MMP3 & & & & & & & & & MMP3 & 54228 & 36 & $2(1)$ \\
\hline MOES & & & & & & & & & MOES & 67892 & 50 & $5(2)$ \\
\hline MYH15 & & & & & MYH15 & 225904 & 43 & $1(1)$ & & & & \\
\hline MY01A & MYO1A & 119238 & 39 & $1(1)$ & & & & & & & & \\
\hline NACA & & & & & NACA & 23370 & 214 & $4(4)$ & NACA & 23370 & 320 & $6(6)$ \\
\hline NACA2 & & & & & NACA2 & 23209 & 104 & $1(1)$ & NACA2 & 23209 & 130 & $2(2)$ \\
\hline NACAD & & & & & & & & & NACAD & 161971 & 66 & $1(1)$ \\
\hline \multicolumn{13}{|l|}{ NFM } \\
\hline NOP2 & & & & & & & & & NOP2 & 89589 & 42 & $1(1)$ \\
\hline NPM & & & & & NPM & 32726 & 65 & $6(3)$ & NPM & 32726 & 597 & $18(12)$ \\
\hline OVCH1 & & & & & OVCH1 & 127095 & 36 & $1(1)$ & & & & \\
\hline PA2G4 & & & & & PA2G4 & 44101 & 35 & $2(1)$ & & & & \\
\hline
\end{tabular}




\begin{tabular}{|c|c|c|c|c|c|c|c|c|c|c|c|c|}
\hline PARK7 & & & & & & & & & PARK7 & 20050 & 36 & $2(1)$ \\
\hline PCBP1 & & & & & & & & & PCBP1 & 37987 & 32 & $1(0)$ \\
\hline PCNP & & & & & & & & & PCNP & 18913 & 37 & $2(1)$ \\
\hline PDIA1 & & & & & PDIA1 & 57480 & 649 & $27(20)$ & PDIA1 & 57480 & 884 & $31(28)$ \\
\hline PDIA4 & & & & & PDIA4 & 73229 & 37 & $1(1)$ & PDIA4 & 73229 & 452 & $22(16)$ \\
\hline PDIA6 & PDIA6 & 48490 & 62 & $2(1)$ & & & & & & & & \\
\hline PERI & PERI & 53651 & 90 & $3(1)$ & & & & & & & & \\
\hline PGK1 & & & & & & & & & PGK1 & 44985 & 78 & $4(2)$ \\
\hline PGK2 & & & & & & & & & PGK2 & 45166 & 79 & $1(1)$ \\
\hline PHB & & & & & $\mathrm{PHB}$ & 29843 & 39 & $2(1)$ & & & & \\
\hline PHLA1 & PHLA1 & 45501 & 35 & $1(1)$ & & & & & & & & \\
\hline PKHG1 & PKHG1 & 156541 & 30 & $1(1)$ & & & & & & & & \\
\hline PKHG5 & & & & & & & & & PKHG5 & 118974 & 36 & $1(1)$ \\
\hline PLCL2 & & & & & & & & & PLCL2 & 127268 & 36 & $1(1)$ \\
\hline POTEE & & & & & POTEE & 122882 & 90 & $2(1)$ & POTEE & 122882 & 256 & $6(5)$ \\
\hline POTEI & & & & & & & & & POTEI & 122858 & 126 & $5(3)$ \\
\hline PPIA & & & & & PPIA & 18229 & 50 & $5(2)$ & PPIA & 18229 & 210 & $8(6)$ \\
\hline PPIB & & & & & PPIB & 23785 & 80 & $2(1)$ & PPIB & 23785 & 271 & $5(2)$ \\
\hline PRDM8 & & & & & & & & & PRDM8 & 72588 & 34 & $1(1)$ \\
\hline PRDX1 & & & & & PRDX1 & 22324 & 117 & $5(4)$ & PRDX1 & 22324 & 137 & $9(6)$ \\
\hline PRDX3 & & & & & PRDX3 & 28017 & 58 & $1(1)$ & PRDX3 & 28017 & 44 & $3(1)$ \\
\hline PRDX4 & & & & & & & & & PRDX4 & 30749 & 39 & $2(1)$ \\
\hline PRDX6 & & & & & PRDX6 & 25133 & 42 & $1(1)$ & PRDX6 & 25133 & 99 & $4(3)$ \\
\hline PROF1 & & & & & PROF1 & 15216 & 75 & $3(2)$ & PROF1 & 15216 & 163 & $5(5)$ \\
\hline PSA3 & & & & & PSA3 & 28643 & 78 & $2(1)$ & PSA3 & 28643 & 172 & $6(6)$ \\
\hline PSIP1 & & & & & & & & & PSIP1 & 60181 & 70 & $3(1)$ \\
\hline PVRL4 & & & & & & & & & PVRL4 & 55933 & 34 & $1(1)$ \\
\hline RAB1A & & & & & & & & & RAB1A & 22891 & 52 & $1(1)$ \\
\hline RAB28 & & & & & RAB28 & 25054 & 36 & $1(1)$ & RAB28 & 25054 & 39 & $1(1)$ \\
\hline RAB35 & & & & & & & & & RAB35 & 23296 & 52 & $1(1)$ \\
\hline RALY & & & & & RALY & 32501 & 66 & $4(1)$ & RALY & 32501 & 111 & $5(5)$ \\
\hline
\end{tabular}




\begin{tabular}{|c|c|c|c|c|c|c|c|c|c|c|c|c|}
\hline RALYL & & & & & RALYL & 32425 & 66 & $1(1)$ & RALYL & 32425 & 71 & $1(1)$ \\
\hline RAN & & & & & RAN & 24579 & 87 & $4(2)$ & RAN & 24579 & 39 & $1(1)$ \\
\hline RBBP4 & RBBP4 & 47911 & 44 & $4(1)$ & & & & & & & & \\
\hline RBBP5 & RBBP5 & 59800 & 33 & $1(0)$ & & & & & & & & \\
\hline RBBP7 & RBBP7 & 48132 & 88 & $3(2)$ & & & & & & & & \\
\hline RL11 & & & & & & & & & RL11 & 20468 & 86 & $2(1)$ \\
\hline RL22 & & & & & RL22 & 14835 & 181 & $4(4)$ & RL22 & 14835 & 157 & $2(2)$ \\
\hline $\mathrm{RL7}$ & & & & & & & & & RL7 & 29264 & 52 & $2(1)$ \\
\hline RL8 & & & & & RL8 & 28235 & 35 & $1(1)$ & & & & \\
\hline RLA0 & & & & & RLAO & 34423 & 111 & $4(3)$ & RLA0 & 34423 & 258 & $6(6)$ \\
\hline RLAOL & & & & & RLAOL & 34514 & 48 & $1(1)$ & & & & \\
\hline RLA1 & & & & & RLA1 & 11621 & 41 & $1(1)$ & & & & \\
\hline RLA2 & & & & & RLA2 & 11658 & 63 & $2(1)$ & RLA2 & 11658 & 206 & $5(5)$ \\
\hline ROA3 & & & & & & & & & ROA3 & 39799 & 34 & $1(1)$ \\
\hline RS14 & & & & & RS14 & 16434 & 35 & $1(1)$ & & & & \\
\hline RS15A & & & & & & & & & RS15A & 14944 & 36 & $1(1)$ \\
\hline RS16 & & & & & RS16 & 16549 & 49 & $1(1)$ & & & & \\
\hline RS28 & & & & & & & & & RS28 & 7893 & 35 & $1(1)$ \\
\hline RS3A & & & & & & & & & RS3A & 30154 & 43 & $4(1)$ \\
\hline RS4X & & & & & RS4X & 29807 & 61 & $1(1)$ & & & & \\
\hline RS8 & & & & & RS8 & 24475 & 85 & $2(2)$ & & & & \\
\hline RU17 & & & & & RU17 & 51583 & 41 & $1(1)$ & & & & \\
\hline SAE2 & & & & & & & & & SAE2 & 71749 & 56 & $3(2)$ \\
\hline SAFB1 & & & & & & & & & SAFB1 & 103036 & 40 & $2(1)$ \\
\hline SARNP & & & & & & & & & SARNP & 23713 & 52 & $3(2)$ \\
\hline sep-02 & & & & & sep-02 & 41689 & 59 & $1(1)$ & sep-02 & 41689 & 149 & $2(2)$ \\
\hline SF3A1 & & & & & SF3A1 & 88888 & 75 & $7(4)$ & SF3A1 & 88888 & 238 & $15(12)$ \\
\hline SF3B2 & & & & & SF3B2 & 100279 & 139 & $3(2)$ & SF3B2 & 100279 & 36 & $1(1)$ \\
\hline SNED1 & & & & & SNED1 & 158206 & 33 & $1(1)$ & SNED1 & 158206 & 36 & $1(1)$ \\
\hline SPTN4 & SPTN4 & 290005 & 42 & $1(1)$ & & & & & & & & \\
\hline SRSF1 & & & & & SRSF1 & 27842 & 57 & $1(1)$ & SRSF1 & 27842 & 109 & $4(2)$ \\
\hline
\end{tabular}




\begin{tabular}{|c|c|c|c|c|c|c|c|c|c|c|c|c|}
\hline SRSF3 & & & & & SRSF3 & 19546 & 91 & $3(3)$ & SRSF3 & 19546 & 53 & $3(1)$ \\
\hline ST134 & & & & & & & & & ST134 & 27561 & 53 & $4(1)$ \\
\hline TAGL2 & & & & & & & & & TAGL2 & 22548 & 56 & $1(1)$ \\
\hline TBA1A & & & & & TBA1A & 50788 & 59 & $1(1)$ & TBA1A & 50788 & 43 & $1(1)$ \\
\hline TBA1B & & & & & TBA1B & 50804 & 368 & $13(11)$ & TBA1B & 50804 & 586 & $22(18)$ \\
\hline TBA1C & TBA1C & 50548 & 363 & 17(10) & & & & & & & & \\
\hline TBA3E & & & & & TBA3E & 50568 & 105 & $6(4)$ & & & & \\
\hline TBA4A & & & & & TBA4A & 50634 & 218 & $9(7)$ & & & & \\
\hline TBA4B & & & & & & & & & TBA4B & 27819 & 202 & $4(4)$ \\
\hline TBA8 & & & & & TBA8 & 50746 & 150 & $7(5)$ & TBA8 & 50746 & 262 & $10(7)$ \\
\hline TBB1 & & & & & TBB1 & 50865 & 148 & $5(4)$ & TBB1 & 50865 & 124 & $7(4)$ \\
\hline TBB2A & & & & & TBB2A & 50274 & 57 & $1(1)$ & TBB2A & 50274 & 599 & $21(14)$ \\
\hline TBB2C & TBB2C & 50255 & 98 & $5(3)$ & & & & & & & & \\
\hline TBB3 & & & & & TBB3 & 50856 & 90 & $2(2)$ & TBB3 & 50856 & 105 & $4(3)$ \\
\hline TBB4B & & & & & TBB4B & 50255 & 566 & $19(14)$ & TBB4B & 50255 & 722 & $25(17)$ \\
\hline TBB5 & & & & & TBB5 & 50095 & 777 & $20(24)$ & TBB5 & 50095 & 936 & $30(21)$ \\
\hline TBB8 & TBB8 & 50257 & 103 & $5(2)$ & & & & & & & & \\
\hline TCP4 & & & & & & & & & TCP4 & 14386 & 139 & $4(1)$ \\
\hline TENS1 & & & & & TENS1 & 186499 & 38 & $1(1)$ & & & & \\
\hline TPIS & & & & & TPIS & 31057 & 75 & $2(1)$ & TPIS & 31057 & 68 & $4(2)$ \\
\hline TRAP1 & & & & & TRAP1 & 80345 & 96 & $2(1)$ & TRAP1 & 80345 & 54 & $1(1)$ \\
\hline TSNA1 & TSNA1 & 56655 & 40 & $1(1)$ & & & & & & & & \\
\hline UN13C & & & & & UN13C & 252693 & 39 & $2(1)$ & & & & \\
\hline UTS2 & & & & & & & & & UTS2 & 14515 & 39 & $1(1)$ \\
\hline VIME & & & & & VIME & 53676 & 100 & $1(1)$ & VIME & 53676 & 344 & $10(8)$ \\
\hline WNT7A & & & & & WNT7A & 40405 & 34 & $1(1)$ & & & & \\
\hline XRCC6 & & & & & & & & & XRCC6 & 70084 & 53 & $4(1)$ \\
\hline ZN275 & ZN275 & 49780 & 34 & $1(1)$ & & & & & & & & \\
\hline ZN524 & & & & & ZN524 & 29262 & 44 & $1(1)$ & & & & \\
\hline
\end{tabular}

Table S9 Phosphoproteins relationship according to which replicates were identified 


\begin{tabular}{|ll|l|l|l|l|}
\hline GO:0000226 & microtubule cytoskeleton organization & 272 & $11(4.0 \%)$ & 0.00402 & 0.0216 \\
\hline GO:0012501 & programmed cell death & 1551 & $38(2.5 \%)$ & 0.0042 & 0.022 \\
\hline GO:0051235 & maintenance of location & 173 & $8(4.6 \%)$ & 0.00619 & 0.0316 \\
\hline GO:0048519 & negative regulation of biological process & 2999 & $63(2.1 \%)$ & 0.00862 & 0.0429 \\
\hline GO:0009611 & response to wounding & 1105 & $28(2.5 \%)$ & 0.00888 & 0.0431 \\
\hline
\end{tabular}

Table S10 Enriched gene ontology level 3 categories of biological processes of identified phosphoproteins. Set size refers to the number of entities that have a UniProt ID as stated in the corresponding GO category at the ConsensusPathDB site. The number of candidates contained refers to amount of proteins identified in this study that appear as part of the GO category. P-values are calculated according to a hypergeometric test; q-values represent $p$-values corrected for multiple testing using the false discovery rate method.

\begin{tabular}{|l|l|l|l|l|}
\hline Pathway name & $\begin{array}{l}\text { Set } \\
\text { size }\end{array}$ & $\begin{array}{l}\text { Candidates } \\
\text { contained }\end{array}$ & P-value & Q-value \\
\hline Signaling events mediated by HDAC Class III & 40 & $17(43.6 \%)$ & $5.03 \mathrm{E}-19$ & $2.57 \mathrm{E}-17$ \\
\hline p38 signaling mediated by MAPKAP kinases & 22 & $7(33.3 \%)$ & $1.45 \mathrm{E}-07$ & $3.38 \mathrm{E}-06$ \\
\hline LKB1 signaling events & 45 & $9(20.9 \%)$ & $1.99 \mathrm{E}-07$ & $3.38 \mathrm{E}-06$ \\
\hline Validated targets of C-MYC transcriptional activation & 87 & $11(13.6 \%)$ & $8.79 \mathrm{E}-07$ & $1.12 \mathrm{E}-05$ \\
\hline Class I PI3K signaling events mediated by Akt & 36 & $7(20.0 \%)$ & $6.55 \mathrm{E}-06$ & $5.64 \mathrm{E}-05$ \\
\hline mTOR signaling pathway & 65 & $9(14.1 \%)$ & $6.64 \mathrm{E}-06$ & $5.64 \mathrm{E}-05$ \\
\hline Insulin-mediated glucose transport & 31 & $6(20.0 \%)$ & $3.07 \mathrm{E}-05$ & 0.000224 \\
\hline FoxO family signaling & 50 & $7(14.3 \%)$ & $6.52 \mathrm{E}-05$ & 0.000416 \\
\hline Trk receptor signaling mediated by PI3K and PLC-gamma & 37 & $6(16.7 \%)$ & $9.07 \mathrm{E}-05$ & 0.000514 \\
\hline $\begin{array}{l}\text { Role of Calcineurin-dependent NFAT signaling in } \\
\text { lymphocytes }\end{array}$ & 57 & $7(13.0 \%)$ & 0.000123 & 0.000576 \\
\hline Signaling events mediated by HDAC Class II & 39 & $6(15.8 \%)$ & 0.000124 & 0.000576 \\
\hline a6b1 and a6b4 Integrin signaling & 46 & $6(13.3 \%)$ & 0.000324 & 0.00138 \\
\hline $\begin{array}{l}\text { Regulation of nuclear beta catenin signaling and target gene } \\
\text { transcription }\end{array}$ & 81 & $7(8.8 \%)$ & 0.0014 & 0.00548 \\
\hline Aurora B signaling & 43 & $5(11.9 \%)$ & 0.00174 & 0.00635 \\
\hline Integrin-linked kinase signaling & 46 & $5(11.1 \%)$ & 0.00238 & 0.00802 \\
\hline HIF-1-alpha transcription factor network & 66 & $6(9.1 \%)$ & 0.00252 & 0.00802 \\
\hline Regulation of Telomerase & 73 & $6(8.8 \%)$ & 0.00293 & 0.00878 \\
\hline Caspase cascade in apoptosis & 53 & $5(9.4 \%)$ & 0.00489 & 0.0138 \\
\hline PDGFR-beta signaling pathway & 130 & $8(6.3 \%)$ & 0.00518 & 0.0139 \\
\hline Aurora C signaling & 7 & $2(33.3 \%)$ & 0.0062 & 0.0158 \\
\hline ATR signaling pathway & 37 & $4(10.8 \%)$ & 0.0072 & 0.0175 \\
\hline
\end{tabular}

Table S11 Enriched PID pathway-based sets of identified phosphoproteins. Set size refers to the number of entities that have a Uniprot ID as stated in the corresponding PID pathway-based set at the ConsensusPathDB site. The number of candidates contained refers to amount of proteins which are part of the extended network and appear as part of the pathway. P-values are calculated according to a hypergeometric test; $q$-values represent $p$-values corrected for multiple testing using the false discovery rate method. 


\begin{tabular}{|l|l|l|l|l|}
\hline Pathway name & $\begin{array}{l}\text { Set } \\
\text { size }\end{array}$ & $\begin{array}{l}\text { Candidates } \\
\text { contained }\end{array}$ & P-value & Q-value \\
\hline Pathogenic Escherichia coli infection & 55 & $16(29.1 \%)$ & $1.16 \mathrm{E}-14$ & $7.33 \mathrm{E}-13$ \\
\hline Systemic lupus erythematosus & 138 & $19(14.0 \%)$ & $5.10 \mathrm{E}-11$ & $1.61 \mathrm{E}-09$ \\
\hline Ribosome & 91 & $14(15.6 \%)$ & $4.66 \mathrm{E}-09$ & $9.79 \mathrm{E}-08$ \\
\hline Gap junction & 89 & $13(14.6 \%)$ & $3.68 \mathrm{E}-08$ & $5.80 \mathrm{E}-07$ \\
\hline Spliceosome & 127 & $15(11.8 \%)$ & $6.07 \mathrm{E}-08$ & $7.65 \mathrm{E}-07$ \\
\hline Glycolysis / Gluconeogenesis & 65 & $11(16.9 \%)$ & $8.96 \mathrm{E}-08$ & $9.41 \mathrm{E}-07$ \\
\hline Alcoholism & 179 & $17(9.5 \%)$ & $2.00 \mathrm{E}-07$ & $1.80 \mathrm{E}-06$ \\
\hline Protein processing in endoplasmic reticulum & 166 & $16(9.6 \%)$ & $3.79 \mathrm{E}-07$ & $2.99 \mathrm{E}-06$ \\
\hline Phagosome & 154 & $15(9.9 \%)$ & $6.56 \mathrm{E}-07$ & $4.59 \mathrm{E}-06$ \\
\hline Antigen processing and presentation & 76 & $10(13.2 \%)$ & $3.85 \mathrm{E}-06$ & $2.43 \mathrm{E}-05$ \\
\hline Legionellosis & 55 & $8(14.5 \%)$ & $1.73 \mathrm{E}-05$ & $9.92 \mathrm{E}-05$ \\
\hline Epstein-Barr virus infection & 203 & $14(7.0 \%)$ & $8.43 \mathrm{E}-05$ & 0.000443 \\
\hline Cell cycle & 124 & $10(8.1 \%)$ & 0.000275 & 0.00133 \\
\hline Pyruvate metabolism & 41 & $5(12.2 \%)$ & 0.00158 & 0.00712 \\
\hline Propanoate metabolism & 32 & $4(12.5 \%)$ & 0.0043 & 0.0181 \\
\hline Phenylalanine metabolism & 18 & $3(16.7 \%)$ & 0.00592 & 0.0233 \\
\hline
\end{tabular}

Table S12 Enriched KEGG pathway-based sets of identified phosphoproteins. Set size refers to the number of entities that have a Uniprot ID as stated in the corresponding KEGG pathway-based set at the ConsensusPathDB site. The number of candidates contained refers to amount of proteins which are part of the extended network and appear as part of the pathway. P-values are calculated according to a hypergeometric test; q-values represent $p$-values corrected for multiple testing using the false discovery rate method. 